Annuaire suisse de politique de développement

2 | 1982

Annuaire Suisse - Tiers Monde 1982

\title{
La Coopération de la Suisse au développement : Mesures économiques et commerciales
}

Wirtschaftliche und Kommerzielle Entwicklungszusammenarbeit

Antoine Brawand

\section{CpenEdition}

1 Journals

Édition électronique

URL : http://journals.openedition.org/aspd/1136

DOI : $10.4000 /$ aspd. 1136

ISSN : 1663-9669

Éditeur

Institut de hautes études internationales et du développement

Édition imprimée

Date de publication : 31 août 1982

Pagination : 207-266

ISSN : $1660-5934$

\section{Référence électronique}

Antoine Brawand, « La Coopération de la Suisse au développement :

Mesures économiques et commerciales ", Annuaire suisse de politique de développement [En ligne], 2 |

1982, mis en ligne le 27 janvier 2013, consulté le 08 septembre 2020. URL : http://

journals.openedition.org/aspd/1136; DOI : https://doi.org/10.4000/aspd.1136 


\title{
LA COOPERATION DE LA SUISSE AU DEVELOPPEMENT : MESURES ECONOMIQUES ET COMMERCIALES
}

\author{
Antoine Brawand
}

\section{WIRTSCHAFTLICHE UND KOMMERZIELLE ENTWICKLUNGSZUSAMMENARBEIT}

Zusammenfassung : Der Verfasser vertritt den Standpunkt, dass die wirtschaftlichen Beziehungen zwischen der Schweiz und der dritten Welt durch ein schwerwiegendes Missverhältnis gekennzeichnet sind. Der Autor zeigt zunächst den wirtschaftlichen Kontext auf und befasst sich dann mit der Einbeziehung der Ziele der Entwicklungszusammenarbeit in das Feld der Wirtschaftspolitik. Seit dem 28. November 1978 gibt es einen Bundesbeschluss, der finanzielle Mittel für wirtschafts- und handelspolitische Massnahmen im Rahmen der internationalen Entwicklungszusammenarbeit freigibt. Von den Ausgaben her gesehen sind hauptsächlich die Mischkredite und die Zahlungsbilanzhilfe in diesen Massnahmen enthalten. Der Verfasser erörtert auch die Fragen der Grundprodukte, der Industrialisierung und der Handelsförderung zugunsten der Entwicklungsländer. Er analysiert die gegenwärtigen Handlungsprinzipien der Eidgenossenschaft im Bereich der Mischkredite, die Darlehensbedingungen, das Nutzungsfeld dieser Kredite und die Wahl der verschiedenen Länder als Empfänger einer solchen Hilfe. Es ist festzustellen, dass die Erteilung der Mischkredite den schweizerischen Aussenhandel dank Finanzierung der Lieferungen unterstützt. Dadurch festigt sich auch die Stellung schweizerischer Firmen auf manchen Handelsplätzen. Zum Abschluss dieser Untersuchung kommt der Verfasser zu dem Ergebnis, dass die Aussenwirtschaftspolitik noch zu wenig die Zielen der Entwicklungszusammenarbeit berücksichtigt. Es geht vor allem darum, die Tätigkeit der Privatunternehmen effektiv in eine für die Entwicklung günstige Richtung zu steuern. 


\section{INTRODUCTION}

La mise en cuvre de la coopération économique et commerciale au développement, partie intégrante de la politique de développement, intervient dans le contexte de la politique économique extérieure. Plusieurs questions se posent, en particulier : la politique de développement agit-elle sur des leviers significatifs de la politique économique extérieure? Les mesures économiques et commerciales sont-elles conformes aux principes de la politique de développement?

Ces questions sont vitales. Avec la crise mondiale, le sort des pays, des régions et des groupes sociaux les plus défavorisés tend à s'aggraver davantage.

$D$ 'autre part, les relations économiques entre la Suisse et le Tiers Monde se caractérisent par des asymétries croissantes.

L'étude présente d'abord le déséquilibre des échanges Suisse-Tiers Monde (I), puis traite de l'insertion des buts de la coopération au développement dans le champ bien protégé de la politique économique extérieure (II). Cinq types de mesures économiques et commerciales sont alors passés en revue : les crédits mixtes, le soutien de la balance des paiements de pays pauvres, l'action en faveur des exportateurs de matières premières, la promotion commerciale des produits du Tiers Monde et la coopération industrielle (III). Le cas des crédits mixtes sera approfondi car il illustre une des tendances actuelles de la coopération internationale au développement : I'association de capitaux publics et de capitaux privés (IV). Certains chapitres et la conclusion contiennent des recommandations concernant le renforcement de la coopération économique et commerciale au développement.

Note : Cette étude doit beaucoup à tous ceux qui ont bien voulu me faire part de leurs critiques et de leurs suggestions. Je leur en suis très rec.onnaissant. II va sans dire que j'assume l'entière responsabilité de la présentation des faits et de leur interprétation. 


\section{LE DESEQUILIBRE DES ECHANGES SUISSE-TIERS MONDE (1)}

Disposant d'un marché intérieur prospère, mais de dimension modeste, la Suisse s'est profondément engagée dans la division internationale du travail. Les principaux courants d'échanges se déroulent avec les pays à économie de marché. En 1981, ces derniers ont livré le $87 \%$ des importations et absorbé le $75 \%$ des exportations suisses. Les échanges avec les pays du Tiers Monde ont représenté $9 \%$ des importations et $21 \%$ des exportations. II est intéressant de les comparer à la part du Tiers Monde dans le commerce extérieur des pays de I'OCDE : $30 \%$ des importations et $26 \%$ des exportations.

La situation géographique et la spécialisation économique de la Suisse représentent des causes évidentes de la faible part des importations en provenance du Tiers Monde. De nombreuses matières premières, notamment presque tous les produits pétroliers raffinés et la plupart des métaux, nous parviennent par I'intermédiaire de pays industrialisés (en particulier nos voisins) où elles ont subi une première transformation. Elles apparaissent donc dans les statistiques comme des importations provenant de ces pays. Ce biais statistique n'explique cependant pas tout. L'industrie suisse, qui se situe généralement en aval des premiers stades du processus de production, utilise relativement peu de matières premières brutes. La constatation de ces causes ne devrait cependant pas nous faire oublier l'existence d'obstacles à la diffusion des produits du Tiers Monde sur les marchés suisses. Nous reviendrons sur ce problème dans l'étude des mesures de promotion commerciale en faveur des pays du Tiers Monde (cf. chap. III).

Sur le plan des exportations, la situation est très différente. Sans atteindre les taux élevés enregistrés en Amérique du Nord (31\%) et au Japon (45\%), la part des pays en développement dans les livraisons suisses $(21 \%)$ dépasse les pourcentages enregistrés par la CEE $(20 \%)$ et surtout par l'AELE $(15 \%)$. Dans la lutte toujours plus dure que les exportateurs se livrent aujourd'hui pour la conquête et le contrôle des débouchés, les marchés du Tiers Monde comptent. Les pays pétroliers et les "nouveaux pays industrialisés" exercent le plus d'attrait. II s'agit d'un nombre restreint de pays.

En 1981, les exportations suisses vers le Tiers Monde se sont élevées à 11,1 milliards de francs et les importations à 5,5 milliards, laissant apparaître un excédent commercial de 5,6 milliards. Si on connaissait l'origine et la destination effectives des marchandises, ce solde serait sensiblement réduit mais il resterait important. L'excédent du commerce Suisse-Tiers Monde contraste fortement avec le déficit global du commerce extérieur suisse. (En 1981, les exportations se sont élevées à 52,8 milliards de francs et les importations à 60,1 milliards, provoquant un déficit de 7,3 milliards.)

En ce qui concerne la rémunération des services (en particulier les primes d'assurance, les frais bancaires, les droits de licence) et les revenus de capitaux, 
la Suisse réalise un grand excédent. Malgré l'absence de ventilation régionale des recettes et des dépenses totales, on peut affirmer que par rapport au volume des affaires traitées avec le Tiers Monde, le solde actif est substantiel.

L'examen des balances du commerce, des services et des revenus de capitaux montre donc un déséquilibre structurel important des transactions courantes entre l'économie suisse et le Tiers Monde. Certes, pour les raisons indiquées plus haut, l'équilibre ne peut pas être atteint sur le plan bilatéral. Cependant, l'excédent en faveur de la Suisse est considérable (2).

La dépendance économique et politique en est une cause générale bien con. nue. Les nations du Tiers Monde subissent une division internationale du travail défavorable à leur développement. La majorité des pays doivent accepter des termes de l'échange inéquitables. Sur le plan interne, la dépendance se traduit par la désarticulation des structures sociales et, pour la majeure partie de la population, par la non couverture des besoins fondamentaux. Bien que ces processus dépassent le seul cadre des relations entre la Suisse et le Tiers Monde, notre pays peut cependant favoriser des changements (3).

La Suisse ne publie toujours pas la balance des mouvements de capitaux. Selon le Mémorandum remis chaque année au Comité d'aide au développement de I'OCDE, I'économie suisse contribue beaucoup au financement des pays en développement. En pourcent du produit national brut, les flux "nets" de capitaux privés en provenance de la Suisse sont les plus élevés des pays membres du CAD. Rappelons cependant que les statistiques du CAD recensent mieux les mouvements de capitaux destinés aux pays en développement que les flux en retour! En outre, la répartition détaillée, par pays d'origine et de destination, n'étant pas divulguée, il n'apparaît pas si les capitaux suisses parviennent effectivement dans les pays qui ont les besoins de financement extérieur les plus grands du point de vue de la politique de développement.

Voici quelques traits significatifs des mouvements de capitaux en provenance de la Suisse :

- Les trois quarts des flux se dirigent vers les pays industrialisés, et moins d'un quart vers les pays en développement. Dans la répartition des investissements directs, la part de ces pays est encore moindre : un dixième de la valeur totale des investissements à l'étranger. Cette différence s'explique également par la situation géographique et I'histoire de la Suisse, ainsi que par la spéculation internationale de son économie. S'appuyant sur les structures d'approvisionnement établies par les autres pays industriels dès la colonisation, l'industrie suisse a relativement peu investi dans les activités extractives et dans l'agriculture (4). Ses investissements se sont concentrés dans les industries de transformation, dans des pays qui offrent des débouchés importants, particulièrement en Amérique latine.

- Au cours de la dernière décennie, les mouvements de capitaux privés ont augmenté très rapidement. Dans la statistique officielle des ressources nettes mises à la disposition des pays en développement, cette croissance 
apparaît clairement. La progression des flux suisses de capitaux privés a été la plus spectaculaire. Les augmentations les plus fortes concernent les investissements de portefeuille, les prêts bancaires et les crédits à l'exportation. (Dès 1980, ces flux ont rapidement baissé, mais ils restent à un niveau élevé.) Les investissements directs ont perdu la première place. Notons qu'une bonne partie des flux de capitaux privés placés par des résidents suisses proviennent du Tiers Monde, notamment des pays pétroliers (mais aussi de pays pauvres!).

L'état actuel des statistiques (caractérisé par le manque ou l'hétérogénéité des données) ne permet pas de savoir dans quelle mesure et à quel prix les placements de capitaux privés compensent l'excédent de recettes que la Suisse réalise dans les opérations courantes avec les pays en développement (commerce, services et revenus de la fortune). De toute façon, les pays qui bénéficient $d^{\prime} u n e$ telle compensation supportent souvent des inconvénients notoires. Les prêts permettent de maintenir ou d'accroître le pouvoir d'achat au prix d'un endettement croissant. Les investissements directs renforcent souvent l'emprise étrangère. Pour pouvoir donner une appréciation complète, il faudrait aussi connaître l'origine et le montant des fuites de capitaux qui aggravent la situation de bien des pays déficitaires.

A cause de son volume restreint, l'aide publique suisse n'a pas d'effet sensible au niveau macroéconomique. Les prestations de la Confédération ont légèrement augmenté en pourcent du produit national brut au cours des années 1970. Cependant, par rapport aux flux financiers privés, elles ont très fortement diminué.

Bien que la majeure partie des prestations fédérales ne soit pas liée à des commandes de biens et de services auprès de fournisseurs suisses, elles ont des répercussions favorables à nos entreprises. Le Conseil fédéral, qui a récemment publié les résultats d'une première enquête menée à ce sujet, écrit : "I'aide publique accordée par la Confédération aux pays en développement s'élevait à 406,2 millions de francs en 1980. Pour la même année, les achats réalisés en Suisse se montaient à 337 millions de francs, qui traduisent l'effet mesurable de cette aide sur l'économie de notre pays". Le Conseil fédéral indique qu'en outre, la valeur des livraisons de biens et de services effectués pour des projets financés par la Banque internationale de reconstruction et de développement (BIRD) s'est élevée à 214 millions de francs (5).

Pour conclure notre brève présentation des relations économiques SuisseTiers Monde, comment les caractériser globalement? Dans les nombreux messages et rapports rédigés à l'appui des demandes de crédits pour la coopération au développement, le Conseil fédéral insiste sur la notion d'interdépendance. La Suisse dépend de ses fournisseurs de matières premières et d'énergie, tandis que le Tiers Monde a besoin de nos produits manufacturés, de nos services et de nos capitaux. L'intensification des échanges répondrait donc, de façon évidente, à des intérêts mutuels. Cette interprétation tient cependans très peu compte de la dépendance des peuples du Tiers Monde envers 
les sociétés industrialisées. Les relations économiques Suisse-Tiers Monde se caractérisent aussi par cette asymétrie fondamentale. Le déséquilibre structurel des opérations courantes en est une manifestation éloquente.

Malgré leur opposition sur le fond de l'analyse, les partisans des deux approches s'entendent sur le fait que le secteur privé joue un rôle prépondérant dans l'évolution des relations économiques Suisse-Tiers Monde. La Confédération s'étant engagée à renforcer la coopération au développement, il convient d'examiner dans quelle mesure elle réussit à associer l'économie privée à la réalisation de cet objectif. 


\section{LA COOPERATION AU DEVELOPPEMENT DANS LE CONTEXTE DE LA POLITIQUE ECONOMIQUE EXTERIEURE}

Le Conseil fédéral considère la coopération au développement "comme un élément important de notre politique étrangère et comme une tâche à remplir dans le contexte de notre politique économique extérieure" (1). Dans le débat qui se poursuit aujourd'hui à ce propos, deux questions notamment sont posées : les objectifs de la politique économique extérieure et ceux de la coopération au développement sont-ils vraiment compatibles? Quels sont les moyens d'action des responsables de la coopération au développement dans le domaine des relations économiques extérieures?

\section{Des objectifs compatibles?}

Les buts de la coopération au développement sont définis par l'article 5 de la loi fédérale sur la coopération au développement et l'aide humanitaire internationales. II s'agit de soutenir les efforts des pays en développement en vue d'améliorer les conditions de vie de leurs populations, de contribuer à mettre ces pays en mesure d'assurer leur développement par leurs propres forces et de tendre, à long terme, vers un meilleur équilibre au sein de la communauté internationale. La Confédération soutient en priorité les efforts des pays en développement, régions et groupes de population les plus défavorisés (2).

Ces objectifs sont-ils compatibles avec ceux de la politique économique extérieure? Le Conseil fédéral en est convaincu. La Suisse s'intéresse aux marchés du Tiers Monde, a besoin de la sécurité de l'approvisionnement et souhaite "un ordre économique mondial de caractère libéral dans toute la mesure du possible" (3). Les pays en développement y trouveront un intérêt réciproque seulement si les pays industrialisés leur accordent un soutien accru pour faire progresser le développement économique et social.

Dans cette perspective, la compatibilité de la politique économique extérieure et de la coopération au développement découle de l'interdépendance (4). “Le renforcement de l'interdépendance économique exprime en fait une dépendance croissante de chaque pays par rapport à l'ensemble de la communauté internationale. Elle conduit à reconnaître la co-responsabilité de tous les Etats à l'égard de leurs partenaires. Seule une coopération toujours plus poussée peut à la fois sauvegarder les intérêts nationaux et promouvoir le développement d'ensemble" (5).

Le Conseil fédéral reconnaît que la politique de développement et la politique economique extérieure "peuvent parfois viser aussi des objectifs qui leur sont propres". Des différences dans l'établissement de priorités peuvent engendrer des tensions qu'une approche pragmatique est seule apte à surmonter (6). 
Qu'est-ce qui distingue exactement les deux politiques? Pour le Conseil fédéral, "la différence réside dans I'accent mis sur tel ou tel aspect : la politique économique extérieure se préoccupe avant tout de la position de la Suisse dans l'ensemble des relations économiques mondiales. II s'agit de la défense d'intérêts suisses légitimes". En revanche, "la politique suisse de développement définit la contribution spécifique que peut apporter notre pays au progrès économique et social des pays en développement et à leur participation à l'économie mondiale, que ce soit directement par des mesures spéciales en faveur de ces pays ou indirectement par l'aménagement des relations économiques en général" (6).

En principe, "ces deux approches sont indissociables et cherchent à atteindre le même but à long terme : la création, sur les plans national et international, de conditions offrant à tous les pays la possibilité de tirer profit de manière équitable de leur participation à l'économie mondiale" (6).

Sur la base de cette définition, on pourrait conclure que les objectifs de la politique de développement et de la politique économique extérieure sont tout à fait compatibles.

\section{Quels moyens d'action?}

Dans sa première demande de crédit concernant le "financement de mesures de politique économique et commerciale au titre de la coopération internationale au développement", le Conseil fédéral a écrit : la loi sur la coopération au développement "nous commande d'apporter à certaines mesures classiques de politique économique et commerciale, de même qu'aux nouvelles mesures que nous entendons mettre en œuvre, des aménagements tels qu'elles puissent mieux répondre à la situation et aux besoins particuliers des pays en développement" (7).

Trois formes de coopération au développement concernent particulièrement la politique économique extérieure. Elles sont définies par l'article 6 de la loi (alinéa 1, lettres b, c et d) :

- L'aide financière (en partie) qui contribue notamment à développer l'infrastructure économique et sociale des pays auxquels elle est destinée.

- Des mesures de politique commerciale qui visent notamment à assurer une meilleure participation des pays en développement au commerce mondial, afin qu'ils puissent en retirer des avantages plus substantiels.

- Des mesures en vue d'encourager l'engagement de ressources du secteur privé, telles que les investissements, de nature à favoriser le développement au sens de l'article 5 (buts de la coopération suisse au développement).

Les instruments qui correspondent à ces trois formes de coopération au développement figurent dans le tableau suivant : 


\section{LA COOPERATION ECONOMIQUE ET COMMERCIALE AU DEVELOPPEMENT}

\section{Mesures financières}

Crédits mixtes * :

Il s'agit de prêts liés à l'achat de marchandises suisses, accordés conjointement par la Confédération et un consortium de banques suisses. La part de la Confédération est accordée pour une longue durée et sans intérêt.

Mesures destinées à soutenir les balances des paiements des pays les plus défavorisés * :

- Prêts bilatéraux à des conditions libérales ou dons.

- Contribution au fonds de bonification d'intérêts du FMI.

Contribution à un fonds de bonification d'intérêts de la Banque mondiale (3ème guichet, ouvert temporairement en 1975-1976) *.

\section{Mesures de politique commerciale}

Stabilisation des prix de certains produits de base et diversification des exportations des pays en développement * :

- Participation au Fonds commun du Programme intégré pour les produits de base.

- Participation à des accords de produits.

- Eventuellement, contribution à des projets bilatéraux.

Promotion commerciale en faveur des pays en développement ${ }^{*}$. (Mesures bilatérales et multilatérales d'encouragement des importations en provenance du Tiers Monde.)

Préférences tarifaires. (Participation de la Suisse au système généralisé de préférences tarifaires en faveur des pays en développement et autres abaissements non réciproques du tarif douanier.)

Mesures propres à encourager l'engagement de ressources du secteur privé

Crédits mixtes * (déjà mentionnés sous "Mesures financières").

Garantie contre les risques de l'investissement (GRI).

Mesures en faveur de l'industrialisation des pays en développement : encouragement des investissements directs et du transfert de technologie ${ }^{*}$. 
Coopération avec l'Organisation des Nations Unies pour le développement industriel (ONUDI) pour faciliter les transferts de technologie et les investissements directs*.

Coopération avec d'autres organisations internationales s'occupant de la définition des conditions juridiques, économiques et sociales de l'investissement à l'étranger dans les pays en développement, en particulier :

- Commission des Nations Unies sur les sociétés transnationales. Participation à l'élaboration du projet de code de conduite, soutien du Centre sur les sociétés transnationales.

- CNUCED : Code de conduite des Nations Unies sur le transfert de technologie.

- Organisation de coopération et de développement économiques (OCDE). Participation aux travaux de l'Organisation et adhésion à la Déclaration sur les investissements internationaux et les entreprises multinationales.

\footnotetext{
Remarque :

La Confédération suisse participe aussi à un grand nombre de conférences internationales concernant simultanément plusieurs formes de coopération. Exemples : Conférence sur la coopération économique internationale (Paris, 1975-77). Comités de l'Assemblée générale des Nations Unies pour la stratégie internationale du développement et pour la préparation de négociations globales, Conférence sur les pays les moins avancés (Paris, septembre 1981), etc.

\begin{abstract}
Note :
Les mesures signalées par un astérisque entraînent des dépenses comprises dans les statistiques de l'aide publique au développement (APD). Elles sont présentées dans les chapitres III et IV.
\end{abstract}

Comment le Conseil fédéral a-t-il défini le champ de la coopération économique et commerciale au développement? Voici ses critères : "Pour autant qu'une mesure soit inspirée avant tout par des considérations de politique de développement, elle prend l'une des formes définies à l'article 6 de la loi fédérale sur la coopération au développement et l'aide humanitaire internationales". Inversement, "les articles 2 (principes) et 5 (objectifs) de la loi s'appliquent aux mesures qui sont essentiellement motivées par des considérations de politique de développement" (8).

Ces critères paraissent clairs, mais le champ de la coopération économique et commerciale ne se laisse pas délimiter aussi nettement. Le Conseil fédéral a parfois assimilé, de façon implicite, les mesures suivantes à des actions de coopération au développement : la garantie fédérale contre les risques à l'exportation, les accords de protection des investissements privés, les accords de double imposition et les mesures tendant à faciliter l'accès des pays en 
développement au marché suisse des capitaux (9). L'application de ces mesures n'est pourtant pas subordonnée aux objectifs de la politique de développement.

Le débat concernant la garantie fédérale contre les risques à l'exportation ( $G R E$ ) a mis en évidence le problème de la définition du champ d'application de la politique de développement. La GRE est le principal instrument fédéral de promotion des exportations. Elle a aussi pour but de maintenir et de créer ainsi des possibilités de travail en Suisse. Plus de la moitié des crédits garantis concerne des exportations à destination des pays en développement. Lorsque les biens vendus sont utiles au développement des pays acheteurs, l'octroi de la GRE répond aussi à leurs intérêts. Cette condition n'est pas toujours remplie mais la loi fédérale sur la GRE n'oblige généralement pas l'autorité d'exécution à en tenir compte. Les exportateurs peuvent donc, avec l'appui de la Confédération, inciter des clients (jugés dignes de crédit) à faire des acquisitions ruineuses pour l'économie de leur pays.

Le Conseil fédéral a pris plusieurs fois position sur cette question. En 1973, il indiquait dans son message à l'appui du projet de loi sur la coopération au développement et l'aide humanitaire internationales : “II ne paraît pas indiqué de lier au présent projet de loi la GRE, puisque cette dernière ne vise pas spécifiquement le développement" (10). Dans des textes plus récents, le Conseil fédéral ou l'administration ont néanmoins rangé la GRE parmi les instruments qui permettent de favoriser le développement du Tiers Monde grâce à l'engagement accru de ressources du secteur privé (9).

De nombreuses voix se sont élevées, en particulier au sein des organisations bénévoles de coopération au développement, des mouvements de solidarité et du Parlement, pour dénoncer l'ambivalence d'une garantie fédérale contre les risques à l'exportation censée servir à la fois les exportateurs suisses et les peuples du Tiers Monde. Le gouvernement chercha à résoudre la contradiction en précisant que la GRE ne faisait pas partie de la politique de développement. Cette réponse ne donna pas satisfaction. Après avoir penché d'abord en faveur de la prise en considération complète des buts de la coopération au développement, le Parlement inséra la disposition suivante dans la loi fédérale sur la garantie contre les risques à l'exportation : "S'agissant d'exportations à destination des pays en développement les plus défavorisés, la Confédération tiendra compte des principes fondamentaux de la politique suisse en matière d'aide au développement" (11).

Quelles sont les conséquences de ce compromis pour l'industrie d'exportation? Le groupe des pays les plus défavorisés (I'administration se basera généralement sur la liste de cinquante-deux pays établie par I'OCDE) compte relativement peu dans les échanges internationaux. II absorbe environ $2,5 \%$ des exportations suisses et bénéficie de $8 \%$ des crédits à l'exportation couverts par la GRE (12). Les chiffres correspondants pour l'ensemble des pays en développement étaient $22 \%$ des exportations suisses en 1981 et $66 \%$ des crédits à l'exportation assurés à la fin de l'année (13). Autrement dit, pour la 
plupart des affaires avec les pays en développement (concernant les neuf dixièmes des exportations et les sept huitièmes des engagements de la GRE), la Confédération n'a toujours pas l'obligation légale de tenir compte des principes fondamentaux de la coopération au développement.

Cet exemple montre comment l'un des principaux instruments de la politique économique extérieure peut rester détaché, pour l'essentiel, des buts de la politique de développement, bien qu'il joue un rôle capital dans le domaine du développement! S'agit-il d'un cas particulier ou sommes-nous en présence d'un phénomène plus large? Cherchant à répondre à cette question, nous avons dressé une liste d'instruments de la politique économique ex térieure (voir ci-dessus) et l'avons confrontée à celle des instruments de la coopération économique et commerciale au développement (voir plus haut).

\section{LA POLITIQUE ECONOMIQUE EXTERIEURE DE LA SUISSE}

Mesures ayant des répercussions importantes sur le développement du Tiers Monde ( $y$ compris mesures indiquées dans le premier tableau)

\section{Mesures concernant plusieurs types d'échanges}

\section{a) Mesures multilatérales}

Participation aux négociations internationales sur les problèmes économiques Nord-Sud (commerce, finances, énergie, technologie, développement, etc.) dans le cadre des institutions spécialisées des Nations Unies, du GATT, de l'OCDE, etc., ainsi que de conférences ad hoc (par exemple Conférence sur la coopération économique internationale, Paris, 1975-77).

Suivi (parfois participation à certaines commissions) des travaux d'organisations internationales dont la Suisse n'est pas membre (Assemblée générale des Nations Unies, Fonds monétaire international, Banque mondiale) et prises de position (la Confédération suisse a par exemple déclaré qu'elle s'associait à la mise en œuvre de la Stratégie internationale du développement pour les années 1980).

Collaboration à des mesures monétaires internationales, en particulier : garantie jusqu'à concurrence de deux milliards de francs accordée par la Confédération à la Banque nationale suisse pour lui permettre de soutenir des opérations à moyen terme du Fonds monétaire international. Contributions à des opérations internationales de soutien de la balance des paiements de pays déficitaires. 


\section{b) Mesures bilatérales}

Missions de "goodwill", échanges de lettres ou conclusion d'accords concernant le développement des échanges.

Commissions mixtes (Suisse-pays partenaire)

\section{c) Mesures autonomes}

Politique d'ouverture aux échanges internationaux dans les secteurs industriels, des services et de la finance; protectionnisme agricole.

Appui de l'administration fédérale et des ambassades aux opérations commerciales et financières privées.

Loi fédérale sur les mesures économiques extérieures (1982) : Permet au Conseil fédéral de prendre mesures défensives au cas où le trafic des marchandises, des services ou des paiements serait affecté par des décisions (protectionnistes notamment) de gouvernements étrangers.

\section{Mesures concernant le commerce}

\section{a) Mesures multilatérales}

Participation au Système généralisé des préférences tarifaires.

Participation au Programme intégré pour les produits de base (Fonds commun et accords de produits).

Participation à l'Agence internationale de l'énergie (institution rattachée à I'OCDE).

Adhésion au code de conduite des Nations Unies sur les pratiques commerciales resctrictives.

Participation à la négociation du code de conduite des Nations Unies sur le transfert de technologie.

Interventions en faveur de la libéralisation des échanges commerciaux (au GATT, à la CNUCED, à l'OCDE, etc.). 


\section{b) Mesures bilatérales}

Accords commerciaux.

Voir aussi point 3 b) (crédits-cadres et crédits mixtes).

\section{c) Mesures autonomes}

Promotion des exportations suisses :

- Garantie fédérale contre les risques à l'exportation (GRE) : voir point $3 \mathrm{c})$.

- Subvention à l'Office suisse d'expansion commerciale (OSEC).

Importations :

- Les produits industriels originaires du Tiers Monde peuvent, en principe, presque tous entrer en franchise (exception pour des textiles, habits, chaussures, etc.). En outre, la Confédération encourage quelques importations.

- Protectionnisme dans le secteur agricole, en général.

- Recherche de la sécurité de l'approvisionnement en matières premières et en énergie.

Surveillance du commerce de certains produits stratégiques.

\section{Mesures concernant les flux de capitaux privés}

\section{a) Mesures multilatérales}

Participation à des négociations internationales sur des opérations de consolidation de dettes.

Participation, dans le cadre de l'OCDE, aux négociations sur les conditions des crédits à l'exportation.

Adhésion à la Déclaration de l'OCDE sur les investissements internationaux et les entreprises multinationales.

Participation à la négociation concernant le code de conduite des Nations Unies sur les firmes transnationales.

\section{b) Mesures bilatérales}

Accords concernant les crédits-cadres ouverts par des banques suisses (couverts par le GRE). 
Accords concernant les crédits mixtes ouverts par la Confédération et par des banques suisses (la part privée est couverte par la GRE).

Accords de consolidation de dettes.

Accords de protection des investissements.

Accords de double imposition.

\section{c) Mesures autonomes}

Garantie fédérale contre les risques à l'exportation (GRE) :

- Risque politique et de non-transfert (y compris de moratoire).

- Risque d'insolvabilité ou de refus de payer de collectivités publiques (risque de ducroire).

- Risque monétaire.

- Risque avant livraison.

Garantie des risques à l'investissement. Encouragement d'investissements de petites et moyennes entreprises dans des pays en développement.

Facilités accordées aux émissions d'obligations des banques internationales de développement (en particulier de la BIRD).

Réglementation libérale des transactions financières internationales (généralement pas de restriction des opérations de change, secret bancaire, etc.).

\section{Principales sources :}

- P. Hammer (1974), G. Berweger (1977), G. Rehsche (1977), Ph. Berberat et H. Stetter (1979), F. Bluntschli (1980).

- Conseil fédéral :

Message relatif à la loi fédérale sur les mesures économiques extérieures du 7.12.81;

Rapports sur la politique économique extérieure.

Les références se trouvent dans la bibliographie.

La comparaison des deux tableaux montre que les instruments suivants, notamment, restent détachés de la politique de développement : la promotion des exportations, les consolidations de dettes, les accords de protection des investissements privés et les règles concernant les transactions financières avec l'étranger. Ces instruments auraient pourtant un grand rôle à jouer dans une politique qui viserait à corriger les principaux déséquilibres structurels des échanges entre la Suisse et le Tiers Monde. 


\section{MESURES ECONOMIQUES ET COMMERCIALES}

Depuis le 28 novembre 1978, un arrêté fédéral prévoit le "financement de mesures de politique économique et commerciale au titre de la coopération internationale au développement" (ci-après : mesures économiques et commerciales) (1). La continuation du financement de ces mesures fait l'objet d'un nouvel arrêté. Le Conseil national l'a adopté le 23 juin 1982. Le Conseil des Etats doit se prononcer en automne 1982.

Les mesures économiques et commerciales correspondent, au même titre que la coopération technique notamment, à des formes d'action prévues par la loi sur la coopération au développement et l'aide humanitaire internationale. Ces formes sont, en l'occurrence, l'aide financière (les crédits mixtes en font partie), des mesures de politique commerciale et des mesures d'encouragement de flux de capitaux privés. La mise en œuvre des mesures, qui intervient dans le contexte de la politique économique extérieure, est soumise aux principes et aux buts de la coopération au développement (voir chap. II). L'Office fédéral des affaires économiques extérieures (OFAEE), du Département fédéral de l'économie publique, est chargé de l'exécution des mesures. La Direction de la coopération au développement et de l'aide humanitaire (DDA), du Département fédéral des affaires étrangères, a le droit de se prononcer. En ce qui concerne les crédits mixtes, I'OFAEE agit d'entente avec la DDA (2).

Nous examinerons d'abord le coût des mesures et les objectifs généraux, puis cinq rubriques correspondant au crédit de programme : crédits mixtes, aide à la balance des paiements, produits de base, promotion commerciale en faveur des pays en développement et industrialisation (3).

\section{Coat des mesures}

Seules les mesures qui entraînent des coûts distincts des dépenses de fonctionnement de l'administration fédérale sont considérées ici. Comme nous I'avons relevé dans le chapitre précédent, le champ de la coopération économique et commerciale au développement est plus large. Nous ne traitons pas non plus d'une mesure relativement importante qui se traduit par une diminution de recettes : les préférences tarifaires. En 1980, ces dernières ont entraîné une moins-value des recettes douanières estimée à 36 millions de francs (4).

Ainsi définies, les dépenses concernant les mesures économiques et commerciales se sont élevées à 7,2 millions de francs en 1979, 17,7 millions en 1980 et 29,2 millions en 1981 (5). Elles ont représenté $2,1 \%$ de l'aide publique au développement en $1979,4,3 \%$ en 1980 et $6,5 \%$ en 1981 . L'augmentation découle de la réalisation des engagements de dépenses effectués sur la base 
de l'arrêté fédéral du 28 novembre 1978. Dans son Message sur la continuation du financement de mesures de politique économique et commerciale, du 14 décembre 1981, le Conseil fédéral indique qu'à l'avenir leur coût annuel correspondra à environ $15 \%$ de l'aide publique au développement (6).

Voici la répartition des montants des deux premiers crédits de programme destinés au financement de mesures économiques et commerciales :

\begin{tabular}{|c|c|c|c|c|c|c|}
\hline \multirow{3}{*}{ Mesures } & \multicolumn{4}{|c|}{ Crédit de 1978} & \multirow{2}{*}{\multicolumn{2}{|c|}{$\begin{array}{c}\text { Crédit de } 1982 \\
\begin{array}{c}\text { Montants prévus } \\
\text { (répartition } \\
\text { indicative) }\end{array}\end{array}$}} \\
\hline & \multicolumn{2}{|c|}{$\begin{array}{l}\text { Montants prévus } \\
\text { (répartition } \\
\text { indicative) }\end{array}$} & \multicolumn{2}{|c|}{$\begin{array}{l}\text { Montants engagés } \\
\text { au 15.9.1982 }\end{array}$} & & \\
\hline & Mio Fr. & $\%$ & Mio Fr. & $\%$ & Mio Fr. & $\%$ \\
\hline Crédits mixtes & 110 & 55,0 & 112,5 & 65,6 & 240 & 68,5 \\
\hline $\begin{array}{l}\text { Aide à la balance des } \\
\text { paiements }\end{array}$ & 50 & 25,0 & 33,0 & 19,2 & 70 & 20,0 \\
\hline Produits de base & 25 & 12,5 & 20,7 & 12,1 & 20 & 5,7 \\
\hline Promotion commerciale & 10 & 5,0 & 2,3 & 1,3 & 10 & 2,9 \\
\hline Industrialisation & 5 & 2,5 & 3,0 & 1,8 & 10 & 2,9 \\
\hline TOTAL & 200 & 100,0 & 171,5 & 100,0 & 350 & 100,0 \\
\hline
\end{tabular}

Sources :

- Messages concernant le financement de mesures de politique économique et commerciale au titre de la coopération internationale au développement, des 9.9.1978 et 14.12.1981 (Montants prévus).

- Renseignements communiqués par l'OFAEE (montants engagés au 15.9.1982).

Considérées sous l'angle des dépenses, les mesures économiques et commerciales comprennent donc principalement des crédits mixtes et l'aide à la balance des paiements. Ensemble, ces deux mesures financières ont absorbé $85 \%$ des engagements de dépenses sur le crédit de programme de 1978 et elles représentent, à titre indicatif, $89 \%$ des dépenses prévues dans le cadre du nouveau crédit de programme. Les montants engagés ou prévus pour les produits de base, la promotion commerciale et l'industrialisation sont en revanche faibles.

La disparité entre les deux groupes de dépenses s'explique en partie par une différence de nature : les crédits mixtes et l'aide à la balance des paiements impliquent toujours des mises de fonds alors que les autres mesures consistent souvent dans l'envoi d'experts, la formation de spécialistes, la création 
de contacts et l'encouragement de l'initiative privée. Le second type d'action requiert davantage de personnel. L'OFAEE se heurte ici, comme la DDA, à un obstacle toujours plus contraignant : le blocage des effectifs du personnel de la Confédération. Cependant, l'examen des mesures nous permettra de constater aussi d'autres causes qui empêchent le lancement d'actions de grande envergure dans des domaines essentiels comme les matières premières, la promotion commerciale et l'industrialisation.

\section{Objectifs généraux}

Le Conseil fédéral écrit que "la coopération économique et commerciale au développement constitue l'un des moyens" d'atteindre les objectifs généraux de la politique de développement. Elle favorise "la participation des pays en développement à l'économie mondiale et aux mécanismes de la coopération économique internationale en général" et leur permet " $d$ 'en tirer le meilleur parti, notamment dans le but de créer les conditions économiques et sociales favorables à l'épanouissement de l'être humain"' (7).

La plupart des mesures de politique économique et commerciale "sont liées à des activités de l'économie suisse, ou ont une incidence sur elles, notamment lorsqu'elles tendent à améliorer le fonctionnement de l'économie mondiale... Elles servent ies intérêts à long terme de notre pays. Certaines d'entre elles auront en outre des effets positifs immédiats sur l'économie suisse. Elles sont donc l'exemple de mesures qui répondent à la fois à nos intérêts et à ceux des pays en développement dans un monde marqué par une interdépendance croissante" (8).

Le Conseil fédéral insiste sur la complexité des mesures économiques et commerciales et indique un certain nombre de limites, en particulier :

- Souvent, les mesures “ne s'appliquent qu'à l'ensemble des pays en développement ou tout au moins à un groupe de ceux-ci. C'est notamment le cas des règles qui régissent le commerce international, comme par exemple les préférences tarifaires. II arrive aussi souvent que des mesures à caractère financier favorisent simultanément des groupes entiers de pays. Les contributions aux accords sur les produits de base (cacao, caoutchouc, etc.) ont pour but de stabiliser les prix d'une matière première déterminée. Lorsque ce but est atteint, le bénéficiaire n'en est pas un seul pays en développement, mais tous les pays exportateurs, qu'il s'agisse d'Etats du Tiers Monde ou de pays industrialisés" (9).

- “L'aide au titre de projets, telle qu'on la trouve dans la coopération technique et dans l'aide financière, a souvent un effet direct sur le milieu ambiant, et, de ce fait, est particulièrement évidente. Les mesures de politique économique et commerciale ont souvent pour but d'améliorer le cadre des échanges économiques internationaux, et de renforcer les économies nationales; elles agissent donc de manière essentiellement indirecte. Elles consti- 
tuent ainsi une des conditions de l'efficacité de mesures qui revêtent plutôt un caractère ponctuel" (9). Notons que les crédits mixtes sont de plus en plus affectés à des projets (même lorsqu'ils consistent en lignes de crédit générales). Cette possibilité existe aussi pour des mesures commerciales, comme nous le verrons plus loin.

_ "Diverses mesures ... supposent certains progrès dans le processus de développement. Par exemple, la promotion des exportations d'un pays ne peut avoir de sens que si celui-ci est décidé à pratiquer une politique encourageant délibérément les exportations, et si dans ce but il produit déjà des marchandises exportables. II en est de même des actions visant I'encouragement des activités du secteur privé : les investissements des entreprises suisses dans un pays en développement nécessitent un climat d'investissement favorable, une certaine infrastructure, ainsi que la possibilité d'écouler les marchandises en faisant un bénéfice" (9).

- Le Conseil fédéral rappelle enfin que, sous notre régime économique, "les échanges commerciaux et financiers avec les pays en développement relèvent pour l'essentiel de l'économie privée" (9). II avait déjà insisté sur ce fait en 1978 : "si la Confédération est à même, par certaines mesures d'encouragement, $d$ 'influer sur les conditions d'une transaction privée, elle ne peut, en règle générale, décider de sa réalisation" (10).

Comme nous l'avons mentionné au début du chapitre II, la Confédération a l'obligation de soutenir en priorité les efforts des pays en développement, régions et groupes de population les plus défavorisés (article 5 de la loi sur la coopération au développement). Comment ceux-ci bénéficient-ils des mesures qui ne leur procurent pas directement certains avantages? Le Conseil fédéral compte sur les effets d'entraînement des activités économiques favorisées à l'aide des mesures. Lorsque la nature de ces dernières permet de choisir les pays bénéficiaires, il convient par conséquent " $d$ 'accorder une attention particulière" à leur politique économique et sociale. "Plus cette politique tend à renforcer la productivité... et à faire participer de larges couches de la population", afin de supprimer les inégalités internes, "plus les mesures d'aide ont des chances d'être couronnées de succès". Le Conseil fédéral rappelle aussi, à ce sujet, "la question des droits de l'homme" (11).

Les mesures économiques et commerciales permettent de soutenir des pays qui ne sont pas encore, pour la plupart, des partenaires importants de la Suisse. Sans intervention publique, ces pays resteraient en marge : "de par leur nature, les efforts de l'économie privée sont essentiellement axés sur les pays et les secteurs qui remplissent les conditions nécessaires pour assurer le succès d'activités orientées vers les affaires" (11). Les interventions de la Confédération tendraient donc à corriger les mécanismes économiques qui ont provoqué, jusqu'à présent, la marginalisation croissante des pays les plus défavorisés.

Enfin, le Conseil fédéral souligne la polyvalence des mesures économiques et 
commerciales. Certaines "sont surtout appropriées pour ceux des pays pauvres qui présentent déjà un revenu par habitant un peu plus élevé. D'autres mesures, de par leur caractère spécifique, sont plus particulièrement adaptées à la situation des pays les plus pauvres" (11). Nous reviendrons sur ce point dans l'examen de chaque mesure.

\section{Crédits mixtes}

Qu'est-ce qu'un crédit mixte? C'est un "financement lié à l'exportation de marchandises ou de services suisses, qui est accordé conjointement par la Confédération (crédit officiel) et par un consortium de banques commerciales suisses (crédit bancaire). La tranche financée par la Confédération correspond à un prêt public à des conditions de faveur, alors que la part financée par les banques constitue un crédit alloué aux conditions du marché. Le résultat de l'opération est un crédit assorti d'un taux d'intérêt moyen et de délais de remboursement prolongés, qui tient mieux compte de la situation du pays en développement importateur" (12).

Pour trois raisons au moins, les crédits mixtes occupent aujourd'hui une place bien visible dans la réflexion sur les relations économiques Suisse - Tiers Monde. Premièrement, comme nous I'avons déjà constaté, la majeure partie du crédit de programme concernant les mesures de politique économique et commerciale est destinée aux crédits mixtes. Deuxièmement, les principaux acteurs de l'économie suisse y participent activement : la Confédération par le biais de mesures financières, les banques par des crédits à l'exportation et I'industrie par des livraisons de biens d'équipement et par la prestation de services. Troisièmement, comme le débat du 23 juin 1982 au Conseil national I'a rappelé, les crédits mixtes font l'objet d'une controverse.

Dans ces conditions, une courte présentation des crédits mixtes n'aurait pas d'intérêt. Nous leur avons par conséquent consacré davantage de place (chapitre IV et annexe).

\section{Aide à la balance des paiements}

Avant d'aborder les mesures spécifiques d'aide à la balance des paiements que la Confédération prend en faveur de pays en développement, il est indispensable de considérer le contexte économique et institutionnel.

Les déficits de la balance des paiements entraînent l'endettement croissant de nombreux pays, en particulier dans le Tiers Monde. Pressés par les échéances, les gouvernements acceptent des conditions de crédit obérantes et compriment les importations de biens essentiels de production et de consommation. Chaque année, plusieurs pays se trouvent pratiquement en cessation de paiement et doivent solliciter de nouveaux délais auprès de leurs créanciers (consolidation de dettes). 
Sur demande des pays débiteurs, le Fonds monétaire international (FMI) accorde certaines facilités financières. Lorsque le besoin de refinancement est important, le FMI subordonne son appui au lancement d'un programme de redressement de l'économie.

En relation ou non avec une consolidation de dettes, les mesures de soutien de la balance des paiements visent à maintenir ou à rétablir la solvabilité des pays déficitaires. Lorsque les revenus de ces derniers sont faibles, une partie des crédits est souvent accordée à des conditions libérales. Elle sert au financement d'importations de biens essentiels (pièces de rechange, produits finis et semi-finis, matières premières, énergie, produits alimentaires, quelques biens d'équipement, etc.). A court terme, ces mesures ont pour but de maintenir la capacité de production et d'atténuer (un peu) les restrictions imposées à la population. A long terme, elles doivent contribuer à la réalisation du programme de redressement économique élaboré par le pays débiteur en collaboration avec les institutions financières internationales.

Bien qu'elle ne soit pas membre du FMI, la Confédération soutient des mesures prises par cette institution. Elle a en particulier accordé une garantie de 2 milliards de francs à la Banque nationale suisse qui peut ainsi contribuer aux opérations à moyen terme du FMI (13). La Banque nationale suisse a notamment participé au financement de la "facilité pétrolière" et, plus récemment, à celui de la "facilité Witteveen" (14). Les pays membres du FMI peuvent y avoir recours lorsque les ressources habituelles du Fonds, attribuées aux pays selon leur quote-part, ne suffisent pas à couvrir des déficits structurels importants de la balance des paiements. Les principaux utilisateurs de ces financements supplémentaires sont actuellement les pays en développement importateurs de pétrole.

Les mesures spécifiques d'aide à la balance des paiements, que la Confédération prend en faveur de pays en développement, sont multilatérales et bilatérales :

- La Confédération a contribué aux comptes de bonification d'intérêts créés dans le cadre des mesures de financement supplémentaires du FMI. L'utilisation de ces comptes permet de réduire le coût des prêts accordés aux pays à bas revenu. En 1975, la Confédération a donné 10 millions de francs pour la "facilité pétrolière" et, en 1981, 5,9 millions de francs pour la "facilité Witteveen".

- Par ses contributions bilatérales, la Confédération participe à des actions coordonnées à l'échelle internationale. La Confédération est en particulier sollicitée lors de l'établissement du programme de redressement consécutif à une consolidation de dettes. La décision doit alors intervenir rapidement. La Confédération a récemment accordé deux dons au Bangladesh (15 millions de francs) et au Soudan (12 millions de francs). Ils sont affectés à des importations destinées aux secteurs clés de l'économie de ces deux pays. Bien que l'aide à la balance des paiements puisse être liée à des 
exportations suisses, les dons faits au Bangladesh et au Soudan ne comportent pas cette obligation. Le don au Bangladesh complète un crédit de programme à l'importation de l'Association internationale de développement (Banque mondiale) et il est géré par elle. En ce qui concerne le Soudan, rappelons qu'en 1979 la Confédération avait fourni un prêt de 16 millions de francs aux conditions du marché dans le cadre d'une consolidation multilatérale de dettes.

Les mesures d'aide à la balance des paiements sont donc principalement destinées à des pays à bas revenus. Les contributions bilatérales vont notamment à des pays pauvres qui ne peuvent pas recevoir de crédit mixte à cause de leur endettement excessif.

Dans l'exposé des motifs, le Conseil fédéral invoque les objectifs de la coopération au développement et montre leur convergence avec ceux de la politique économique extérieure. " L'aide à la balance des paiements a pour but d'assurer aux pays en développement, dans le cadre de mesures coordonnées au niveau international, le maintien de leur capacité d'importation. Le financement des importations de biens essentiels ... contribue à une meilleure utilisation des capacités de production existantes. En même temps, il permet à ces pays d'introduire des mesures de politique économique propres à supprimer les déséquilibres de leur balance des paiements et à rétablir des échanges commerciaux et financiers normaux. Il correspond à l'intérêt qu'a notre pays au maintien de marchés ouverts et de systèmes de paiements stables" (15).

L'utilité de l'aide à la balance des paiements n'est généralement pas contestée. Les modalités et le contexte des interventions sont en revanche fortement critiqués. L'aide à la balance des paiements apparaît souvent comme un édulcorant de la potion amère servie aux nations surendettées.

Fondamentalement, c'est la politique du FMI et celle des principaux bailleurs de fonds qui est mise en cause. Les programmes de redressement économique sont imposés aux pays déficitaires et ils provoquent un vaste transfert de ressources de l'économie intérieure vers le secteur d'exportation. Le coût social est élevé. Enfin, le FMI ne mobilise pas assez de moyens en faveur du Tiers Monde. II ne fait surtout pas assez de prêts à des conditions libérales.

Une politique de coopération financière plus active de la Confédération lui permettrait de moins dépendre de décisions prises ailleurs. Par rapport à l'ensemble des prêts consentis (aux conditions du marché) au titre de la collaboration suisse aux mesures monétaires internationales, l'aide à la balance des paiements consentie à des pays en développement est minime. Elle devrait donc être accrue (de même que les autres composantes de l'aide publique au développement). La Confédération pourrait, en particulier, intervenir davantage sur le plan bilatéral. Par exemple, lorsqu'un pays prioritaire pour les prestations de coopération technique éprouve des difficultés de la balance des paiements, il devrait obtenir facilement des prêts ou des dons supplémentaires de la Suisse. 
Il ne faut pas, pour autant, négliger la coopération avec les institutions financières internationales. Elle est généralement souhaitable car le manque de ressources, en particulier de ressources assorties de conditions libérales, est un facteur indéniable de la sévérité de certains programmes de redressement économique. Au cours des négociations avec le FMI concernant la contribution suisse, la Confédération devrait insister pour que ces programmes concordent le plus possible avec les objectifs de la politique de développement.

\section{Produits de base (16)}

Les pays en développement sont encore très souvent tributaires des exportations d'un nombre réduit de produits primaires. Cette situation freine l'expansion du commerce entre eux, affaiblit leur pouvoir de négociation et leur impose des termes de l'échange défavorables. En outre, les produits primaires étant généralement vendus à l'état brut ou sommairement transformés, la valeur ajoutée sur les lieux de production est faible. A cause des fluctuations de prix, les recettes d'exportation sont incertaines. Cette dépendance gêne la réalisation des programmes de développement.

Les "nouveaux pays industrialisés" ont réussi à modifier sensiblement les modalités de leur insertion dans la division internationale du travail et les pays pétroliers ont obtenu une revalorisation importante de leurs prix. Leur situation reste cependant souvent précaire. Les autres pays en développement dépendent aujourd'hui, dans une large mesure, des résultats des négociations internationales sur les produits de base.

Les pays du Sud revendiquent la revalorisation de leurs exportations de matières premières et, pour compenser l'inflation, l'indexation des cours sur les prix des produits manufacturés. Ils espèrent ainsi parvenir à améliorer le niveau de vie des populations et à financer les importations indispensables à l'industrialisation. Leurs revendications se heurtent au refus du Nord. Celui-ci accepte seulement de favoriser la stabilisation des recettes et des prix des matières premières, et de soutenir des projets pour la mise en valeur des produits. Le premier type d'action ne concerne pas seulement les échanges avec les pays en développement alors que le second contribue, en général, directement aux efforts de ces derniers.

Les principales mesures de stabilisation des recettes ont été instituées par le Fonds monétaire international et par la Communauté économique européenne. Lorsqu'un pays financièrement faible subit une contraction substantielle du montant de ses exportations de matières premières, notamment à cause d'une baisse des cours, il peut obtenir des prêts au titre du mécanisme de compensation du FMI. S'il est associé à la CEE (signataire de la Convention de Lomé), il peut aussi recevoir, en ce qui concerne essentiellement les produits agricoles, des versements compensatoires grâce au système "STABEX". La CEE ne demande pas de remboursement aux pays les plus pauvres. La 
Suisse n'étant ni membre du FMI, ni de la CEE, elle ne contribue pas à ces mécanismes compensatoires. Cependant, l'administration fédérale recherche des modalités de participation. (Concernant le FMI, I'adhésion de la Suisse à cette institution règlerait la question.)

En ce qui concerne la stabilisation des prix, les principaux instruments sont les accords de produit entre pays producteurs et pays consommateurs. Ils comportent, selon les cas, des stocks régulateurs (étain, cacao, sucre, caoutchouc) et des mesures de réglementation des exportations (étain, sucre, café). Les accords internationaux de produit ne sont pas conçus pour corriger l'évolution des prix à long terme. Les pays producteurs et consommateurs ont seulement pu s'entendre sur la limitation des fluctuations conjoncturelles (dues à l'abondance ou à la médiocrité des récoltes par exemple) et, dans quelques cas, sur des contingents d'exportation. Ces objectifs pourtant modestes n'ont, le plus souvent, pas été atteints, à cause de l'insuffisance des moyens d'intervention prévus et du manque de coopération entre les différentes parties. Dans la mesure où celles-ci s'en tiennent aux objectifs de régularisation des cours, la Confédération est favorable au renforcement des accords de produit. Elle a adhéré à la plupart d'entre eux.

A la suite d'une résolution adoptée lors de la IVème session de la CNUCED à Nairobi en 1976, le Nord et le Sud sont parvenus à s'entendre sur la création d'un Fonds commun pour les produits de base (Genève, juin 1980). Ce Fonds doit constituer l'élément central de financement des accords de produit. II comportera deux comptes financièrement indépendants. Le premier disposera d'un capital de $\mathbf{4 0 0}$ millions de dollars. Il contribuera au financement des stocks régulateurs (assuré conjointement par les pays producteurs et consommateurs). Le second compte, doté d'un capital initial de 350 millions de dollars, servira principalement à financer la mise en valeur de matières premières qui ne se prêtent pas à la constitution de stocks régulateurs : il s'agira de projets de transformation des produits, de diversification, de commercialisation, etc. destinés essentiellement aux pays en développement.

Ce résultat est assez éloigné de la résolution adoptée en 1976 par la CNUCED, relative au programme intégré pour les produits de base. Disposant de moyens trop modestes et dépendant de la conclusion d'accords de produit entre producteurs et consommateurs, le Fonds commun n'aura, au début du moins, qu'une influence très réduite sur le commerce international des produits de base. Son action dépendra aussi de la structure des marchés souvent dominés par un nombre réduit d'opérateurs. L'entrée en vigueur de l'accord portant création du Fonds commun n'est en outre pas encore acquise (17).

Le Conseil fédéral a décidé en mai 1982 de ratifier l'accord sur le Fonds commun. La contribution ordinaire de la Suisse a été fixée à 4,35 millions de dollars. La Confédération a en outre accepté de verser une contribution volontaire de 6 millions de dollars pour le second compte.

Les mesures de stabilisation des recettes et des prix, ainsi que de mise en 
valeur des matières premières, présentées plus haut, se situent au plan multilatéral. Les revendications des pays du Tiers Monde touchent aussi les relations bilatérales. Ils souhaitent en particulier conclure des accords de livraison et d'achat à long terme, à des prix rémunérateurs. La Confédération n'entend pas s'engager sur cette voie à cause du volume restreint des importations suisses de matières premières et du rôle subsidiaire de l'Etat suisse (18). Par contre, elle a l'intention de soutenir des projets de commercialisation de matières premières.

La politique suisse dans le domaine des produits de base répond en premier lieu aux objectifs de la politique économique extérieure. II s'agit d'assurer la sécurité de l'approvisionnement à des conditions adaptées aux besoins de l'économie suisse. Les accords de produit doivent atténuer les variations excessives des prix sans en fausser l'évolution à moyen et à long termes. La Confédération espère que ces accords créeront des conditions favorables à l'exploitation des richesses naturelles et qu'ils préviendront la formation de cartels de producteurs, les embargos à l'exportation et toute autre mesure unilatérale qui affecterait particulièrement la Suisse, pays pauvre en matières premières.

Sous l'angle de la coopération au développement, la Confédération entend donner aux pays du Tiers Monde des assurances concernant les possibilités et les conditions d'écoulement des matières premières. Elle souhaite aussi “supprimer progressivement la dépendance des pays en développement visà-vis de structures de production étroites et donc vulnérables en les aidant à mettre sur pied leurs propres industries de transformation" (19). L'action bilatérale et multilatérale de la Confédération favoriserait donc un meilleur équilibre au sein de la communauté internationale et aiderait les pays du Tiers Monde à assurer leur développement par leurs propres moyens, conformément aux objectifs de la loi sur la coopération au développement.

La coût annuel moyen des mesures de la Confédération en faveur des produits de base pourrait s'élever ces prochaines années à environ 7 millions de francs, si l'accord sur le Fonds commun entre en vigueur (20). Ce chiffre montre la modestie des moyens prévus. II ne faut pas se faire d'illusions sur les effets possibles. Notons également que les mesures représentent une condition nécessaire, mais non suffisante, de l'amélioration de la situation des régions et des groupes de population les plus pauvres dans les pays exportateurs de matières premières. Les politiques de développement poursuivies par ces pays joueront un rôle déterminant. Seuls certains projets concrets de transformation et de commercialisation des matières premières peuvent bénéficier directement aux plus pauvres.

La Confédération devrait intensifier son action en faveur des produits de base. Sur le plan multilatéral, elle devrait adhérer aux systèmes existants de stabilisation des recettes, appuyer toute démarche qui faciliterait le démarrage rapide des opérations du Fonds commun et intervenir auprès des autres pays consommateurs pour la conclusion de nouveaux accords de produit. 
Sur le plan bilatéral, deux types de mesures nous semblent indispensables : favoriser, dans la mesure du possible, la conclusion de contrats d'approvisionnement à long terme et à des prix rémunérateurs avec les pays en développement (21), d'une part, et contribuer à la réalisation de projets importants de transformation, de diversification et de commercialisation des matières premières, d'autre part.

\section{Promotion commerciale en faveur des pays en développement}

Hormis les pays pétroliers et les "nouveaux pays industrialisés", la part du Tiers Monde dans le commerce mondial a fortement reculé au cours des trente dernières années. La diminution relative ayant surtout affecté les exportations, les balances commerciales de la plupart des pays en développement se sont soldées par des déficits croissants.

Le déséquilibre les relations commerciales entre la Suisse et le Tiers Monde contribue à ce processus. Nous avons indiqué plus haut quelques causes de la faible part des importations en provenance du Tiers Monde (voir chap. I), par exemple les barrières à l'entrée du marché suisse. Celles-ci s'opposent surtout à la pénétration des biens de consommation et de certains biens intermédiaires. On peut distinguer trois groupes d'obstacles (22) :

- Les tarifs douaniers : ils ne représentent pas une barrière importante. En adhérant au système généralisé de préférences tarifaires, la Suisse a exonéré très largement les importations de produits industriels en provenance de la plupart des pays en développement. Ceux-ci sont ainsi presque traités sur pied d'égalité avec les pays membres de l'Association européenne de libreéchange et de la Communauté économique européenne. Pour différentes raisons, à cause des complications administratives notamment, les préférences tarifaires sont seulement utilisées en partie (23).

- Les obstacles non tarifaires érigés par l'administration publique, en particulier les prescriptions techniques ou sanitaires et les formalités douanières.

- Les obstacles informels érigés par le secteur privé. Ils sont nombreux et divers. On peut citer notamment les exigences suisses concernant la qualité des produits, leur présentation, l'emballage, les modalités de transport et les délais de livraison, le manque d'information des exportateurs du Tiers Monde concernant les usages commerciaux dans les pays industrialisés et les méthodes de vente, ainsi que le manque de soutien de la part des Etats du Tiers Monde dans les domaines des communications, du contrôle de la qualité, de la promotion commerciale, etc. (24).

La suppression ou la réduction des tarifs douaniers bénéficie seulement aux fournisseurs qui surmontent les deux autres groupes d'obstacles. Dans le but de contribuer activement à la croissance de certaines importations, la Confédération a entrepris, au titre de la coopération au développement, la réalisa- 
tion de mesures de promotion commerciale. Elles comportent deux types d'interventions :

- Des tâches permanentes : diffuser des informations sur le marché suisse, fournir des adresses, publier des offres, favoriser des contacts, etc.

- Des actions ponctuelles : réaliser des études de marché, publier du matériel documentaire, inviter des pays en développement à des foires commerciales ou à des expositions, former des spécialistes. Les exportateurs du Tiers Monde apprennent ainsi à maîtriser les méthodes de vente et à adapter leurs marchandises à la demande. Les actions ponctuelles visent souvent des produits et/ou des pays déterminés.

Pour l'exécution des tâches permanentes, la Confédération verse une contribution supplémentaire à I'Office suisse d'expansion commerciale (OSEC). Elle confie les actions ponctuelles à des organismes internationaux et nationaux (25). Depuis 1979, la Confédération a engagé, en tout, des dépenses de 2,3 millions de francs pour la promotion commerciale (état au 15 septembre 1982). Dans le cadre du nouveau crédit de programme, les engagements de dépenses pouvaient s'élever jusqu'à 3 ou 4 millions de francs par an, en moyenne (26).

Les buts présentés par le Conseil fédéral ont surtout trait à la politique de développement. La promotion commerciale est indispensable à l'expansion de l'industrie d'exportation des pays en développement. Elle favorise la diversification de la production et permet de gagner des devises. Elle fait bénéficier les pays du Tiers Monde de la politique commerciale libérale de la Suisse. Les interventions de la Confédération se justifient aussi du point de vue de la politique économique extérieure : les exportations suisses à destination du Tiers Monde ne pourront en effet pas toujours croître plus rapidement que les importations.

Lors du choix des projets, le Conseil fédéral se fonde en outre sur les critères suivants :

- Les bénéficiaires des mesures de promotion commerciale seront des pays à revenu faible ou moyen (PNB inférieur à 1.000 dollars par habitant) possédant une capacité d'exportation suffisante.

- Les mesures serviront à promouvoir des produits d'exportation qui ne sont pas encore compétitifs sur les marchés internationaux. Les ventes auront un effet net positif sur la balance des paiements et la production sera adaptée aux conditions socio-économiques des pays bénéficiaires. L'emploi et la formation professionnelle seront normalement favorisés.

- Les mesures compléteront l'action des milieux économiques. Elles n'exerceront pas d'effets négatifs sur l'ensemble d'une branche économique en Suisse. Elles ouvriront de nouveaux débouchés et créneaux de distribution aux pays bénéficiaires.

Les principales critiques faites aux mesures de promotion commerciale con- 
cernent la conception du développement, la persistance de barrières aux importations et le choix des pays bénéficiaires.

Dans les Messages sur les mesures de politique économique et commerciale, l'ouverture aux échanges et la croissance du commerce extérieur apparaissent souvent comme synonymes de développement. Pourtant, sans une bonne articulation des structures internes, I'insertion des pays du Tiers Monde dans l'économie mondiale accroît leur dépendance. Ils ne peuvent se développer par leurs propres forces (c'est le but de la coopération suisse) qu'en subordonnant leur ouverture aux échanges internationaux à leurs propres objectifs économiques et sociaux.

Malgré l'adoption de préférences tarifaires non réciproques, les produits manufacturés continuent généralement de circuler plus facilement du Nord vers le Sud que dans le sens inverse. La correction de ce facteur de déséquilibre nécessite l'institution d'échanges privilégiés entre les pays du Sud et l'ouverture effective des marchés du Nord. Cette réforme doit être réalisée progressivement afin de faciliter les reconversions nécessaires. Elle devrait être subordonnée au respect de conventions internationales (existantes ou à élaborer) concernant les conditions de travail, la protection de l'environnement et le soutien des industries d'exportation.

L'accès au marché suisse est relativement difficile pour les pays en développement. Il faut donc poursuivre la politique d'ouverture en facilitant l'utilisation des préférences tarifaires et, surtout, en réduisant les barrières non tarifaires et les obstacles informels. Cet objectif comprend la simplification de certaines règles administratives, la circulation de l'information et une concertation permanente entre les exportateurs du Tiers Monde, la Confédération et les importateurs suisses. L'exécution de cette tâche devrait faire partie du travail ordinaire de I'OSEC (la subvention pourrait être augmentée dans ce but) ou être confiée à un service spécialisé.

La poursuite de l'ouverture du marché suisse permettrait d'entreprendre une promotion commerciale plus efficace en faveur des pays en développement. Les moyens d'action actuels devraient être fortement accrus. Les critères de choix des pays bénéficiaires devraient être précisés. Il s'agit de stimuler les exportations de pays qui cherchent à augmenter leurs recettes en devises pour se procurer des biens nécessaires au renforcement de l'économie intérieure. La production des biens exportés doit elle-même exercer des effets favorables sur l'économie des pays fournisseurs.

La création d'un centre de promotion des importations permettrait non seulement de réduire les obstacles à l'importation de produits du Tiers Monde, mais faciliterait une promotion commerciale active et sélective. Ce centre pourrait, le cas échéant, être rattaché à I'OSEC qui reçoit déjà une contribution fédérale pour l'exécution de certaines tâches dans ce domaine. L'action du centre ne devrait toutefois pas être à la remorque de la promotion des exportations! II devrait avoir un cahier des charges précis et collaborer avec 
les instances concernées : organisations internationales, administration fédérale (OFAEE et DDA), économie privée, organisations bénévoles, etc. (27).

La mission du centre serait aussi de participer à la promotion du commerce entre les pays en développement, par la mise à disposition de moyens financiers, d'experts et d'informations (28).

\section{Industrialisation}

L'économie suisse est très active dans la vente d'équipements industriels, I'investissement direct à l'étranger et l'exportation de technologie. Les firmes suisses s'intéressent particulièrement à l'industrie de transformation dans des pays à revenu supérieur ou intermédiaire. En ce qui concerne les pays en développement, plus de $80 \%$ des investissements directs ont eu lieu, en 1979, dans des pays dont le revenu par habitant dépassait 1.000 dollars (29). Ce phénomène de concentration se produit aussi pour les ventes d'équipements industriels et la cession de licences de fabrication.

La Confédération cherche à créer des conditions favorables à ces activités. Ses interventions font essentiellement partie de la politique économique extérieure. Nous en considérerons quelques aspects avant d'examiner les mesures spécifiques de coopération au développement.

Sur le plan multilatéral, relevons la participation de la Confédération à l'élaboration des codes de conduite des Nations Unies sur les firmes transnationales et le transfert de technologie. Selon le Conseil fédéral, l'établissement de nouvelles règles devrait contribuer à promouvoir dans le Tiers Monde des conditions économiques et juridiques favorables à l'engagement des ressources privées du Nord. Le développement du Tiers Monde serait ainsi stimulé.

Sur le plan bilatéral, la Confédération a conclu une série d'accords de protection des investissements (ou comportant, outre des dispositions sur le commerce et la coopération technique, des clauses relatives aux investissements) (30). Au 30 juin 1982, trente-quatre accords avaient été conclus. Les plus grands destinataires des investissements directs suisses (en particulier le Brésil, le Mexique, l'Argentine et l'Inde) ne sont pas du nombre. Les accords ont plutôt pour but de favoriser les investissements dans de nouvelles régions.

La Confédération accorde aussi des garanties contre les risques à l'exportation et à l'investissement (GRE et GRI). La première ne couvre pas seulement des livraisons de biens mais également des prestations de services, en particulier la cession de licences de fabrication (31). La seconde permet aux investisseurs dans les pays en développement de s'assurer contre les risques de nationalisation, d'expropriation, de destruction et de non-transfert de leurs avoirs dans des pays en développement. La GRI a été conçue pour les petites et moyennes entreprises. Elle est accordée pour des investissements dont la capacité de contribuer au développement a été reconnue. Les montants couverts par la GRI sont faibles (32). 
Examinons maintenant les mesures spécifiques de coopération au développement émargeant au crédit de programme concernant les mesures économiques et commerciales.

La Confédération encourage les investissements directs et le transfert de technologie avec le concours de l'Organisation des Nations Unies pour le Développement Industriel (O.N.U.D.I.). La Confédération finance, pour un montant de 400.000 francs environ, un bureau de promotion de la coopération industrielle, ouvert en 1978 à Zurich. Ce bureau est chargé d'identifier des projets dans les pays en développement et de rechercher des investisseurs potentiels. La Confédération lui a aussi attribué un fonds de 500.000 francs destiné au financement d'études de préinvestissement. Les projets choisis doivent associer des petites et moyennes entreprises suisses à des entrepreneurs du Tiers Monde.

Il est prévu d'intensifier ces mesures d'encouragement direct. La Confédération envisage depuis plusieurs années de promouvoir la fondation d'une Société suisse de financement du développement. Celle-ci participerait à la préparation, au financement et à la gestion de projets industriels dans le Tiers Monde.

Enfin, la Confédération subventionne la participation du Tiers Monde à des expositions industrielles. Trois instituts de recherche ont ainsi pu présenter leurs produits à la foire "Technology for the People" à Genève en 1980 et à Mexico en 1981.

Dans I'exposé des motifs concernant la coopération industrielle, le Conseil fédéral donne les arguments suivants. L'industrialisation permet aux pays du Tiers Monde de renforcer leurs structures économiques, d'accroître la part de valeur ajoutée des produits exportés, d'augmenter et de diversifier les recettes en devises. Lorsqu'on utilise des techniques appropriées, la production industrielle procure de l'emploi, contribue à satisfaire les besoins fondamentaux et favorise un processus de développement autonome et cumulatif. II est important de mettre l'expérience et le potentiel de connaissances que l'industrie suisse a accumulés au service de l'économie privée des pays du Tiers Monde.

Lors du débat sur le nouveau crédit de programme concernant les mesures économiques et commerciales, la coopération industrielle est restée à l'arrièreplan. Ce fait s'explique par la modestie des réalisations passées et des dépenses prévues (en tout environ 10 millions de francs pour les prochaines années). Face au dynamisme des grandes firmes dans le domaine de l'investissement direct et du transfert de technologie, les mesures officielles d'encouragement ne sont-elles pas dérisoires? Elles visent, en fait, des acteurs qui tentent de survivre dans la tourmente économique : les petites et moyennes entreprises suisses et les pays en développement dépourvus d'industries compétitives.

Les encouragements de la Confédération peuvent certes faciliter la réalisation de quelques projets intéressants. A cause de leur dimension limitée, ces projets 
n'auront toutefois pas d'impact sensible sur l'industrialisation des pays concernés. Les mesures fédérales méritent néanmoins d'être renforcées. Elles favoriseraient ainsi une certaine diversification de l'offre suisse et permettraient aux petites et moyennes entreprises de répondre à des demandes de coopération industrielle qui $n$ 'intéressent pas les grandes firmes.

Cependant, si le Conseil fédéral entend réellement promouvoir l'industrialisation du Tiers Monde et permettre aux pays en développement de s'insérer favorablement au sein de la division internationale du travail, il devra réunir les moyens de sa politique. II ne s'agit pas d'augmenter considérablement les dépenses publiques. En effet, dans notre pays, la vente d'équipements industriels, l'investissement direct et le transfert de technologie sont essentiellement des activités du secteur privé. Les entreprises ont leurs propres critères de décision, mais elles doivent se conformer à certaines règles d'intérêt général, Il appartient à la Confédération d'expliciter ces règles dans le domaine de la coopération au développement et, en l'occurrence, dans celui de la coopération industrielle.

La tâche est difficile car les intérêts particuliers en jeu sont considérables. De nouvelles possibilités d'action apparaissent aujourd'hui avec l'établissement de codes de conduite internationaux concernant les firmes transnationales et le transfert de technologie. L'élaboration de ces textes n'est certes pas terminée, mais le Tiers Monde a clairement manifesté sa revendication : I'instauration, sur le plan de l'économie internationale, de règles du jeu qui lui permettront de s'industrialiser en profondeur. Une tâche urgente de la coopération au développement est de préparer les instruments juridiques et administratifs nécessaires à la mise en œuvre sur le plan national des codes internationaux de conduite. 


\section{L'ASSOCIATION DE CAPITAUX PUBLICS ET DE CAPITAUX PRIVES : LE CAS DES CREDITS MIXTES}

Les crédits mixtes représentent aujourd'hui la principale mesure de coopération économique et commerciale (voir. chap. III, rubriques "Coût des mesures" et "Crédits mixtes"). Les principaux acteurs de l'économie suisse y participent : la Confédération par le biais de mesures financières, les banques par des crédits à l'exportation et l'industrie par des livraisons de biens d'équipement et la prestation de services.

Avant d'examiner la politique actuelle de la Confédération, il faut considérer le contexte économique - celui des crédits-cadres à l'exportation - iet rappeler succinctement le rôle des premiers crédits mixtes, maintenant épuisés, qui avaient été ouverts essentiellement pour des motifs commerciaux. Nous analyserons ensuite les principes actuels de l'action de la Confédération, les conditions de prêt, le champ d'utilisation des crédits et le choix des pays bénéficiaires. Ces différents éléments permettront de mesurer le chemin parcouru. Nous examinerons aussi les effets des crédits mixtes sur les exportations de biens d'équipement. Pour terminer, nous suggérerons quelques améliorations qui permettraient de souligner l'appartenance des crédits mixtes à la politique de développement.

Le lecteur trouvera en annexe la liste des crédits mixtes accordés à ce jour et des précisions concernant certains points du texte.

\section{Origine des crédits mixtes}

Une partie des prêts à l'exportation consentis par les banques le sont sur la base d'accords généraux appelés "crédits-cadres" ou "crédits de transfert". Ces accords prévoient des conditions uniformes pour toute une série de financements et un montant global. Lors d'accords-cadres avec les pays en développement, la Confédération octroie généralement aux fournisseurs le taux maximal de la garantie contre les risques à l'exportation (95\%).

On peut distinguer trois types d'accords-cadres (1) :

- L'accord de crédit entre une banque et un consortium bancaire et une société étrangère ou un Etat. Les prêts reposent sur une base privée. Les exportateurs doivent néanmoins s'assurer contre les risques à l'exportation. Exemples : les banques suisses ont récemment accordé de telles lignes de crédit (ou en ont prolongé le délai d'utilisation) à des banques algériennes, bulgare, portugaises et de Taïwan.

- L'accord de crédit entre un consortium bancaire et un pays étranger (généralement du Tiers Monde), reposant sur un contrat interétatique. Le premier du genre fut conclu avec I'Inde, en 1960 (2). II prévoyait des 
délais de remboursement de dix ans (au lieu des cinq ans autorisés par le "Gentlemen Agreement" de l'Union des assureurs des crédits internationaux). Exemples récents : accords avec I'Indonésie, la Malaisie, les Philippines et la Corée du Sud.

- L'accord de crédit mixte, enfin, conclu entre un consortium bancaire et un pays en développement d'une part, et entre ce pays et la Confédération d'autre part. Le crédit bancaire est consenti aux conditions du marché tandis que le prêt officiel est alloué aux conditions de l'aide publique au développement. Le premier crédit mixte fut également ouvert à I'Inde, en 1966. Exemples récents : voir la liste figurant en annexe, point 1.

Le consortium bancaire comprend généralement le Crédit Suisse, la Société de Banque Suisse, I'Union de Banques Suisses (I'une des trois est le chef de file) et la Banque Populaire Suisse. Ce cercle s'est élargi depuis quelques années à la Banque Leu S.A. et aux banques cantonales (représentées actuellement par la Banque Cantonale de Berne). Au moment de l'entrée en vigueur de l'accord de crédit, le consortium bancaire diffuse une "note explicative aux exportateurs" indiquant les conditions principales du crédit, les modalités d'utilisation, la procédure d'autorisation, les démarches à entreprendre pour obtenir la garantie contre les risques à l'exportation, etc.

Au moment de la conclusion du premier accord de crédit (1960), les banques suisses ne s'intéressaient pas à l'octroi de prêts importants et de longue durée à des pays relativement peu sûrs. La Confédération parvint à lever les réticences des banques en accordant la couverture maximale prévue par la loi sur la garantie contre les risques à l'exportation (à l'époque $85 \%$ ). Pour sa part, elle refusa, pendant quelques années encore, d'avancer des fonds officiels. Les autorités fédérales estimaient qu'il incombait au secteur privé, en l'occurrence à l'industrie et aux banques, d'accorder des facilités financières aux partenaires commerciaux en difficulté (3).

Cependant, la Confédération finit par donner suite aux demandes répétées de deux pays (I'Inde et le Pakistan) (4). Leurs démarches bénéficiaient de l'appui des exportateurs suisses et des pressions exercées par les grands pays industrialisés. Ceux-ci avaient accordé des. prêts importants à des conditions préférentielles dans le cadre des consortiums d'aide au développement créés sous l'égide de la Banque mondiale. Certaines modalités des premiers crédits mixtes reflètent très clairement l'influence des intérêts économiques suisses, en particulier :

- la répartition du volume des livraisons entre différents secteurs de l'industrie suisse des machines (une tranche fut également réservée pour les "joint-ventures"). La répartition fut opérée sur la base des propositions faites par les gouvernements indien et pakistanais et après entente avec I'industrie des machines;

- des arrangements parallèles ayant pour but de faciliter les échanges économiques (I'Inde avait déjà signé en 1960 une "déclaration de bonnes inten- 
tions" concernant l'importation de montres suisses).

Bien que la Suisse n'ait pas accordé, à proprement parler, des crédits mixtes à la Turquie, il convient de rappeler aussi les prêts à ce pays. La Suisse est membre du consortium d'aide à la Turquie depuis 1962, date de sa création sous l'égide de I'OCDE. En 1964, la Confédération fournit sa première contribution consortiale. Elle comprenait notamment des crédits de longue durée et à taux d'intérêt réduit liés à l'achat de biens d'équipement suisses. En 1968 , un groupe de banques ouvrit un crédit de transfert important. De nouveaux crédits de la Confédération (toujours liés à des exportations suisses) lui succédèrent en 1970 et 1973 (5).

Des conditions de politique économique furent aussi attachées à ces prêts. Le Conseil fédéral le déclara très franchement. En 1968, après avoir critiqué sévèrement le protectionnisme turc, il indiqua : "Bien que l'aide consortiale en faveur de la Turquie ne doive pas, en principe, viser les mêmes buts qu'une négociation commerciale, il va de soi qu'elle implique des efforts de la part de ce pays pour aplanir, dans la mesure du possible, les obstacles existants dans le secteur commercial et sur le plan des investissements". Le Conseil fédéral subordonna l'ouverture de nouveaux crédits au respect de cette condition (6).

Le seul élément permettant de distinguer clairement les premiers crédits mixtes des autres accords de transfert consistait dans les conditions financières libérales de la tranche officielle. La Confédération fit ce geste pour permettre la poursuite des exportations Ses décisions furent motivées essentiellement par des considérations de politique économique extérieure.

Qu'en est-il aujourd'hui ? Que sont devenus les crédits mixtes ?

\section{Principes}

Dans ses exposés des motifs, le Conseil fédéral insiste aujourd'hui sur les objectifs de la politique de développement. Les crédits mixtes permettent de mobiliser des ressources privées supplémentaires pour le développement du Tiers Monde. Ils complètent les autres formes d'aide financière qui sont destinées de plus en plus à ceux des pays pauvres dont la structure économique est encore peu diversifiée. Les crédits mixtes servent à financer des exportations pour la réalisation de projets ou de programmes d'investissement prioritaires dans les plans de développement.

Le Conseil fédéral mentionne aussi la politique économique extérieure. Sur ce plan, les crédits mixtes représentent un instrument de promotion des exportations. Le gouvernement souligne que presque tous les pays industrialisés disposent d'un instrument de ce type. De nombreux pays usent en sus de crédits à l'exportation fortement subventionnés pour accroître la compétitivité de leurs entreprises sur le marché international. En accordant des crédits à des conditions très libérales, la Confédération permet donc à l'indus- 
trie de participer directement à la concurrence qui résulte de la mise en œuvre de cette forme de financement des exportations.

Voici quelques traits marquants de la politique actuelle en matière de crédits mixtes :

- Octroi d'une dizaine ou d'une douzaine de prêts par période de trois à quatre ans.

- Application (compte tenu du caractère spécifique des crédits mixtes) des critères de la politique de développement lors de la définition du champ d'utilisation, du choix des pays et lors de chaque livraison.

- Indication explicite des objectifs de la politique de développement et de la politique économique extérieure.

Les accords de crédits sont conclus selon le principe des avantages mutuels. Le préambule contient généralement la phrase suivante (la formulation précise varie selon les circonstances) : "Le Gouvernement de la Confédération suisse et le Gouvernement de ..., soucieux d'encourager les progrès sociaux et économiques de ..., et également de promouvoir les importations de biens d'équipement et services suisses ... sont convenus de ce qui suit" (viennent alors les articles de l'accord) (7).

Les crédits mixtes font l'objet d'un débat animé. Voici quelques questions soulevées le 23 juin 1982 par des membres du Conseil national, lors de l'examen des mesures économiques et commerciales (8) :

- En liant les crédits à l'achat de biens et de services suisses, ne mélange-ton pas la promotion des exportations et l'aide au développement? "Si notre économie a besoin d'une aide à l'exportation, celle-ci peut se faire de manière indépendante" estime le Groupe socialiste du Conseil national (9). Les partisans des crédits liés affirment par contre que si la Suisse renonçait à cette pratique, les autres pays industrialisés en profiteraient pour nous ravir des marchés. En favorisant la vente de produits suisses, I'on crée ou l'on maintient des emplois en Suisse.

- Cette forme de crédit permet-elle de poursuivre en priorité les objectifs de la politique de développement? Les rapporteurs de la Commission des affaires économiques du Conseil national en sont persuadés. Ils ajoutent qu'une collaboration étroite avec les banques garantit un contrôle efficace des opérations (10). Pour certains orateurs, les crédits mixtes ont même un grand avantage par rapport aux prestations à fonds perdus : on sait où va l'argent (11)!

- Est-il opportun d'augmenter la part des crédits mixtes dans I'aide publique au développement? Selon un rapporteur de la Commission des affaires économiques, les crédits mixtes permettent de mobiliser des ressources privées qui, sans cet encouragement, ne s'investiraient jamais dans le Tiers Monde, en tout cas pas dans des projets prioritaires pour la politique de développement (12). Quant au Groupe socialiste, il demande des garanties : 
le coût des mesures économiques et commerciales ne devra pas dépasser $15 \%$ de l'aide publique au développement (part prévue par le Conseil fédéral) et l'Assemblée fédérale devra être informée des résultats des évaluations concernant les crédits mixtes (9).

On peut résumer le débat en distinguant schématiquement deux tendances. Selon la première, le développement du Tiers Monde et la promotion des intérêts économiques suisse ne doivent pas être mélangés. Selon la seconde, les crédits mixtes permettent de faire d'une pierre deux coups : contribuer au développement du Tiers Monde et donner du travail aux entreprises suisses.

\section{Conditions financières}

Depuis I'ouverture des premiers crédits mixtes, les conditions financières consenties aux pays emprunteurs se sont améliorées :

- Part de la Confédération : la suppression totale des intérêts et l'allongement de la durée des prêts (de quinze ou dix-huit ans à vingt ou vingt-cinq ans) ont accru l'élément de libéralité (13).

- Part du consortium bancaire : comme pour les autres crédits à l'exportation, les banques ont légèrement réduit leur marge et accepté de fixer le taux d'intérêt pour une longue période.

L'élément de libéralité est entièrement à la charge de la Confédération. La réduction de la marge bancaire découle en partie de l'accroissement du taux de la garantie contre les risques à l'exportation.

Les conditions financières des crédits mixtes se situent donc à un niveau intermédiaire entre celles de l'aide publique au développement et celles du marché. Le niveau précis dépend du taux de la participation fédérale. Lors de la fixation de cette dernière, le gouvernement tient compte de la capacité financière des emprunteurs. Jusqu'à présent, la part publique a varié entre $25 \%$ et $50 \%$ du montant total des crédits mixtes. Afin de mieux différencier ceux-ci des crédits à l'exportation ordinaires, la Confédération supportera désormais au moins un tiers des crédits mixtes (14).

Pour pouvoir faire face aux échéances de remboursement (en particulier de la part bancaire), les pays débiteurs ont besoin d'un volume correspondant de recettes extérieures ou d'un allègement de leurs dépenses. II faut donc que les équipements financés à l'aide des crédits mixtes ou le développement d'autres secteurs de l'économie nationale leur permettent d'y parvenir. Pour améliorer simultanément la situation des populations ou des régions les plus pauvres, ces pays doivent maîtriser relativement bien leur processus de développement (15). Nous reviendrons sur ce point dans l'examen du choix des pays bénéficiaires et à la fin du chapitre. 


\section{Champ d'utilisation}

L'Office fédéral des affaires économiques extérieures a établi, en accord avec les banques, une liste standard des biens et services pouvant être financés par les crédits mixtes. Elle est annexée à tous les accords concernant l'ouverture d'une ligne de crédit générale ou limitée à une branche économique prioritaire du pays bénéficiaire. Elle figure aussi dans "les notes explicatives aux exportateurs" diffusées par le consortium bancaire.

Le financement de tout contrat d'achat est subordonné à son acceptation, dans un certain délai, par les autorités chargées de l'exécution de l'accord de crédit. Dans le pays emprunteur, il s'agit par exemple du Ministère du Plan. En Suisse, il faut l'approbation de l'Office fédéral des affaires économiques extérieures et du consortium bancaire. (Celui-ci sollicite d'abord la garantie fédérale contre les risques à l'exportation.)

La Confédération veut s'assurer que l'utilisation des crédits mixtes est conforme aux objectifs de la politique de développement. L'Office fédéral des affaires économiques extérieures est chargé d'appliquer notamment les procédures suivantes :

- Au cours des négocations avec le pays emprunteur, on précise le champ d'utilisation du crédit : projets de développement, services publics, création, extention ou modernisation d'entreprises, infrastructure économique et sociale, etc. Au moment de la signature de l'accord, une série de projets doit normalement être prévue.

- Le lancement d'appels d'offres pour des livraisons importantes ou la consultation d'experts doit assurer la compétitivité des prestations suisses. En ce qui concerne la participation au financement d'un projet unique (par exemple la réalisation d'une centrale hydroélectrique au Honduras ou le montage de locomotives électriques au Zimbabwe), l'octroi des crédits mixtes a suivi la sélection d'entreprises suisses dans le cadre d'un appel d'offres international.

Ces procédures permettent de contrôler l'utilité des prestations suisses et de minimiser les inconvénients inhérents aux crédits liés. Comme pour d'autres types d'interventions, seule la publication du résultat des évaluations intermédiaires et finales en démontrera la conformité aux objectifs de la politique de développement.

Après avoir analysé les conditions financières, I'affectation et les effets socioéconomiques des crédits mixtes, les évaluateurs devraient également estimer les avantages que l'économie suisse en retire : expansion des échanges, concessions éventuelles du pays emprunteur dans d'autres négociations avec la Confédération ou avec des firmes suisses. 
Le Conseil fédéral affirme que les crédits mixtes sont en principe accordés à des pays à bas revenu ayant une capacité d'absorption suffisante, qui poursuivent une politique de développement favorable aux régions et aux populations les plus pauvres. II doit s'agir de pays recherchant des équipements et des services que l'industrie suisse peut vendre à des prix compétitifs (voir description de ces critères en annexe, au point 2.c.).

Une politique suisse de développement mieux structurée qu'autrefois (mais aux moyens toujours insuffisants) permet d'adapter la forme des prestations fédérales à la situation des destinataires. Le Conseil fédéral estime que les contributions aux pays les plus défavorisés doivent être versées sous la forme de dons (16). Lors du choix des destinataires de crédits mixtes, la Confédération tient notamment compte des versements faits au titre de l'aide à la balance des paiements, de l'aide financière et de la coopération technique. D'autres éléments interviennent dans le choix des pays :

- Les demandes des pays en développement eux-mêmes. Ils exigent des crédits à taux d'intérêt réduit et de longue durée en contrepartie de leurs commandes de biens d'équipement.

- Les demandes des banques internationales de développement qui assurent le financement de grands projets d'infrastructure. Les bénéficiaires de commandes importantes sont souvent priés de fournir des crédits à des conditions libérales. La Confédération est par conséquent sollicitée quand des entreprises suisses se qualifient à la suite d'appels d'offres internationaux. L'ouverture de crédits mixtes répond alors à deux objectifs supplémentaires : soutenir l'industrie d'exportation et cofinancer les projets des banques internationales de développement (17).

- Les demandes de l'industrie suisse, appuyées par un consortium bancaire, qui recherche des subventions et des garanties officielles pour s'implanter ou se maintenir sur certains marchés. Les crédits mixtes, qui font l'objet d'accords interétatiques, contiennent ces deux éléments. Sans crédits mixtes, la garantie fédérale contre les risques à l'exportation ne pourrait d'ailleurs parfois pas être obtenue, en tout cas pas au taux maximal de couverture prévu par la loi.

Le choix des pays n'est donc pas simple. Les décisions doivent satisfaires les objectifs de la coopération au développement, respecter les principes de la politique étrangère et tenir compte des intérêts économiques suisses. Précisons ici que ce problème ne touche pas seulement les crédits mixtes : il se pose toujours, avec plus ou moins d'acuité, lors du choix des partenaires de la coopération au développement.

L'octroi controversé d'un crédit mixte au Maroc illustre bien ce problème. Des mouvements de solidarité avec le Tiers Monde, des organisations bénévoles de coopération au développement, des parlementaires, ainsi que des 
journaux ont dénoncé la guerre menée par ce pays, ses dépenses militaires, la compression des prestations sociales, les exportations alimentaires alors qu'une partie de la population est sous-alimentée, etc. Le choix du Maroc ne contredit-il pas les principes de la politique suisse de développement? Au cours du débat sur les mesures économiques et commerciales, plusieurs conseillers nationaux demandèrent au Conseil fédéral de se montrer plus critique dans le choix des pays destinataires de l'aide publique suisse (18).

\section{L'encouragement des exportations}

L'octroi de crédits mixtes favorise les exportations suisses grâce au financement de livraisons (effet direct) et grâce au renforcement de la présence des firmes suisses sur certains marchés (effet indirect).

Le Conseil fédéral estime que "les commandes financées par les crédits mixtes constituent une demande supplémentaire". En effet, "les bénéficiaires du crédit sont bien souvent des pays qui ne pourraient réaliser les projets en cours sans une aide financière extérieure". Le montant annuel des livraisons financées à l'aide des crédits mixtes devrait s'élever, pour les cinq prochaines années, à quelque soixante millions de francs (parts publiques et privées) (19). Comparée au commerce extérieur enregistré en 1981, cette somme représente $0,11 \%$ des exportations totales de la Suisse ou $0,5 \%$ des exportations suisses vers le Tiers Monde (20). Sur le plan global, l'encouragement des exportations est donc minime.

Cependant, il faut aussi considérer l'effet sectoriel. Les ventes de biens d'équipement représentent environ $80 \%$ des exportations de biens et de services financées par des crédits mixtes. On peut donc prévoir que les livraisons annuelles de biens d'équipement bénéficiant de ce mode de financement s'élèveront en moyenne à environ 48 millions de francs pendant ces prochaines années. Ce montant représente $0,27 \%$ des exportations totales de biens d'équipement réalisées en 1981 ou 1,2\% des exportations vers le Tiers Monde. En ne comptant pas les pays en développement principaux exportateurs de pétrole ou d'articles manufacturés (car ils ne reçoivent pas de crédits mixtes), ces livraisons représentent 3,2\% des exportations de biens d'équipement (20).

Nous n'avons pas la place nécessaire à une analyse détaillée des effets sur les exportations vers chaque pays emprunteur. II est néanmoins intéressant de tenter une comparaison générale entre les crédits mixtes et les livraisons actuelles aux pays emprunteurs. La période de déboursement des crédits ne devrait généralement pas durer plus de sept ans. En calculant, pour chaque pays, le rapport entre le montant annuel moyen des déboursements prévisibles et la moyenne des exportations de biens d'équipement enregistrées en 1980-1981, on obtient les pourcentages suivants : 


$\begin{array}{lrlr}\text { Egypte } & 4 & \text { Maroc } & 25 \\ \text { Kenya } & 12 & \text { Zimbabwe } & 28 \\ \text { Pérou } & 13 & \text { Sri Lanka } & 61 \\ \text { Tunisie } & 16 & \text { Sénégal } & 185 \\ \text { Thaïlande } & 16 & \text { Honduras } & 264\end{array}$

Cameroun 20

Moyenne pondérée : $14 \%$ (sans l'Egypte et le Honduras : $20 \%$ ).

Le lecteur trouvera les données et l'explication des calculs en annexe (point 3). Trois indications méritent toutefois de figurer ici même :

- Les crédits mixtes accordés au Honduras et, pour une partie, au Zimbabwe (premier accord) ont facilité l'obtention de commandes à la suite d'un appel d'offres international lancé pour des projets de grande envergure.

- Les pourcentages les plus élevés concernent des pays qui achètent actuellement peu de biens d'équipement en Suisse.

- L'Egypte (pourcentage le plus bas) achète, à elle seule, presque autant de biens d'équipement suisses que les autres pays réunis.

Dans l'ensemble, l'effet d'entraînement des crédits mixtes est modéré. La plupart des crédits accordés ces dernières années favorisent la pénétration de marchés encore restreints. Cependant, ceux-ci pourraient jouer un rôle accru dans le futur.

\section{Souligner l'appartenance à la politique de développement}

La promulgation de la loi sur la coopération au développement a favorisé des changements significatifs dans le domaine des crédits mixtes. Les principes de la politique fédérale sont maintenant mieux définis, les conditions financières ont été améliorées et la sélection des demandes tient principalement compte des buts de la politique de développement.

Les objectifs de la politique économique extérieure et ceux de la politique de développement sont étroitement imbriqués. En soi, ce phénomène est normal car la coopération au développement n'est pas un secteur à part, détaché de l'activité économique. Seules des mesures tendant à orienter celle-ci vers le développement du Tiers Monde sont aptes à corriger progressivement le déséquilibre des relations Suisse-Tiers Monde. Cependant, l'imbrication des objectifs paraît ambiguë si les avantages que les régions et les populations les plus défavorisées doivent retirer des interventions de l'Etat n'apparaissent pas clairement. L'administration fédérale devrait donc publier le plus rapidement possible les résultats d'évaluations concernant l'utilisation des crédits mixtes. 
Comme pour d'autres formes de la coopération au développement, le choix des pays bénéficiaires est délicat. Les buts fixés auront une chance d'être atteints si l'octroi des crédits complète des efforts locaux visant la construction d'un processus de développement viable, la réduction des inégalités sociales et la couverture des besoins fondamentaux de la population. La Confédération doit aussi veiller, en toute logique, à ce que l'ensemble des relations économiques que la Suisse entretient avec ces pays favorisent cette politique.

Les avantages que les pays emprunteurs retirent d'un crédit mixte dépendent également de la qualité des prestations privées. Comme nous l'avons indiqué (à l'annexe, point 2), les intérêts de la tranche bancaire sont généralement fixés de $1 \quad 1 / 2$ à $15 / 8 \%$ au-dessus de celui des obligations de caisse à cinq ou huit ans. Les banques devraient réduire sensiblement leur marge. La sécurité des prêts est presque totale (grâce à la garantie contre les risques à l'exportation) et la gestion est simplifiée (par la fixation de montants minimaux pour les contrats et pour les paiements partiels).

Les pays en développement devraient aussi pouvoir bénéficier des meilleurs prix pour leurs acquisitions de biens et de services. Au sein des organisations internationales, la Suisse s'est prononcée en faveur du déliement de l'aide financière. En ce qui concerne les crédits mixtes, elle soutient les efforts entrepris au sein de l'OCDE pour soumettre les projets de développement importants à des appels d'offres internationaux (21). Cependant, le déliement généralisé des crédits à l'exportation assortis de conditions non commerciales n'est pas pour demain.

Dans cette situation, la Suisse ne devrait-elle pas prendre l'initiative et faire un geste unilatéral? Les principes du libéralisme économique, qui inspirent la conception des interventions de la Confédération, seraient ainsi mieux respectés. La Suisse a-t-elle raison d'y déroger parce que les autres pays industrialisés " $n$ 'ont aucune vergogne à lier leur propre aide à des prestations de leurs entreprises respectives dans une bien plus grande proportion que la Suisse" (22) ? Certains pays étrangers n'ont pas toujours les moyens d'accorder des prestations non liées. Par contre, la Suisse enregistre de grands excédents dans ses paiements courants avec le Tiers Monde. Rappelons aussi que par rapport aux exportations totales de biens d'équipement et de services, le montant des crédits mixtes est modeste. Les commandes qu'ils favorisent ne peuvent pas assurer l'avenir de notre industrie. Celui-ci dépend d'une politique industrielle efficace.

La Confédération a déjà amélioré la procédure d'utilisation des crédits mixtes pour permettre aux emprunteurs de bénéficier de prix plus avantageux. Les grandes commandes résultent souvent d'adjudications internationales. D'autres progrès nous semblent également possibles :

- Le contrôle des règles d'origine pourrait être assoupli, afin d'admettre la participation d'un plus grand nombre de sous-traitants étrangers.

- Une part substantielle de biens et de services étrangers devrait être autori- 
sée pour des projets de développement, lorsqu'elle permet de réduire les coûts ou de procurer des prestations plus adaptées aux besoins.

- Les pays emprunteurs devraient pouvoir affecter une partie des crédits à des livraisons locales (23) ou en provenance d'autres pays en développement (au moins de pays qui ont conclu un accord de coopération économique avec le pays considéré).

De telles améliorations ne nuiraient pas à l'industrie suisse : la politique actuelle consiste déjà à utiliser les crédits mixtes seulement lorsque l'offre suisse est compétitive. En revanche, elles souligneraient, comme les autres aménagements suggérés plus haut, l'appartenance des crédits mixtes à la politique de développement. 


\section{CONCLUSION}

La coopération économique et commerciale au développement s'inscrit dans le contexte d'un grave déséquilibre des rapports Nord-Sud et, en particulier, des échanges entre notre pays et le Tiers Monde. Conformément à la loi sur la coopération au développement, l'action de la Confédération a pour but d'améliorer les conditions de vie des populations du Tiers Monde et de contribuer à mettre les pays bénéficiaires en mesure de se développer par leurs propres forces. A long terme, la coopération doit ainsi favoriser l'établissement de relations équilibrées au sein de la communauté internationale.

Au cours de ces dernières années, la Confédération a renforcé son action. Cependant, les buts retenus dans la loi sur la coopération au développement paraissent de plus en plus éloignés. Dans la confrontation économique mondiale avivée par la crise, la situation des pays, des régions et des groupes de population les plus défavorisés a généralement empiré. Les mesures prises par la Confédération ont peu d'impact sur ce processus. Ce fait ne s'explique pas seulement par la petite dimension de la Suisse. II tient aussi aux limites imparties à l'action des pouvoirs publics.

L'efficacité de la coopération économique et commerciale dépend au moins de quatre facteurs :

- l'importance des moyens d'action de la Confédération,

- la conformité des mesures fédérales aux objectifs de la politique de développement,

- l'orientation de la politique économique extérieure, et

- l'influence sur les activités du secteur privé.

Comme dans les autres domaines de la coopération au développement, la Confédération ne peut pas lancer des actions de grande envergure. En ce qui concerne les cinq types de mesures économiques et commerciales que nous avons analysés, les moyens manquent particulièrement pour les produits de base, l'encouragement des importations et la coopération industrielle. La situation est aggravée par le blocage des effectifs de l'administration fédérale. La timidité de l'action publique contraste avec la croissance rapide des exportations et des transactions financières privées.

Les principes de la coopération économique et commerciale nous paraissent conformes aux objectifs de la politique suisse de développement. Certes, les mesures bénéficient le plus aux pays pauvres ayant déjà une certaine place dans le commerce mondial (avec un revenu toutefois inférieur à 1.000 dollars par habitant). Compte tenu de la prospérité de la Suisse et du déséquilibre de ses échanges avec le Tiers Monde, ces pays ont indiscutablement droit au soutien de la Confédération. II est indispensable de renforcer leur infrastructure économique et d'améliorer les conditions de leur participation aux échanges internationaux. Dans le choix des actions bilatérales, la Confé- 
dération devrait très clairement privilégier les pays qui s'efforcent de poursuivre des politiques de développement favorables aux régions et aux couches sociales les plus pauvres.

Sur le plan de la politique économique extérieure, il faudrait réellement tenir compte des intérêts du Tiers Monde. Des interventions publiques importantes continuent à se dérouler indépendamment des buts de la coopération au développement. Lors des consolidations de dettes, par exemple, la Confédération défend exclusivement les intérêts économiques suisses. Par ailleurs, elle accepte pourtant d'aider quelques pays à rééquilibrer leur balance des paiements. D'autre part, les garanties contre les risques à l'exportation sont accordées (sauf celles qui concernent les pays les plus pauvres) sans aucun examen de la compatibilité des livraisons prévues avec les objectifs de la politique de développement.

Enfin, en ce qui concerne l'activité du secteur privé, la Confédération intervient ponctuellement pour encourager le transfert vers le Tiers Monde de ressources susceptibles de favoriser le développement. Sauf pour les crédits mixtes, les moyens d'action de l'Etat sont très limités. L'encouragement de la coopération industrielle s'adresse par exemple essentiellement aux petites et moyennes entreprises. Le déséquilibre des échanges entre la Suisse et le Tiers Monde continue de croître.

Les objectifs de la politique de développement ne seront pas atteints à l'intérieur des limites actuellement imparties à l'action des pouvoirs publics. Les moyens devraient par conséquent être adaptés aux objectifs.

D'une part, il faut augmenter l'aide publique au développement malgré les restrictions budgétaires. En ce qui concerne les mesures économiques et commerciales, il s'agit de combler les retards accumulés dans les domaines suivants : matières premières, promotion des importations et coopération industrielle. Ce rattrapage nécessite du personnel supplémentaire.

D'autre part, l'efficacité des prestations fédérales pourrait être accrue par la recherche d'une réelle convergence entre la politique de développement et la politique économique extérieure : les intérêts du Tiers Monde doivent être pris en considération dans les décisions concernant notamment la promotion des exportations, les consolidations de dettes, la protection des investissements privés, le transfert de technologie et les transactions financières.

Les interventions fédérales doivent contribuer à orienter les activités économiques privées dans un sens favorable au développement. Même si elle pouvait augmenter considérablement ses prestations, la Confédération ne pourrait pas, à elle seule, remédier au déséquilibre des échanges entre la Suisse et le Tiers Monde. Les entreprises privées peuvent compter sur le soutien de la Confédération dans leurs opérations à l'étranger. II faut qu'à son tour, le comportement des firmes facilite la réalisation des objectifs de la Confédéra- 
tion dans le domaine du développement.

En renforçant ainsi la coopération économique et commerciale au développement, la Suisse honorerait son engagement de participer à la réalisation de la Stratégie internationale du développement pour les années quatre-vingt. 
ANNEXE :

PRECISIONS CONCERNANT LE CHAPITRE IV

Liste des crédits mixtes accordés à ce jour et précisions concernant les conditions financières, le champ d'utilisation, le choix des pays et l'effet sur les exportations suisses de biens d'équipement.

1. Crédits mixtes accordés à ce jour (état au 15 septembre 1982)

\begin{tabular}{|c|c|c|c|c|c|c|}
\hline \multirow[b]{2}{*}{ 离 } & \multirow[b]{2}{*}{$\begin{array}{c}\text { Pays } \\
\text { Type de crédit (1) } \\
\text { Champ d'utilisation }\end{array}$} & \multicolumn{4}{|c|}{ Montants en millions de francs } & \multirow{2}{*}{ 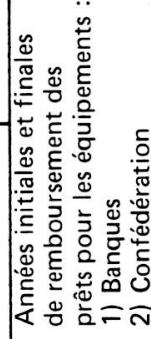 } \\
\hline & & 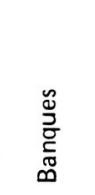 & 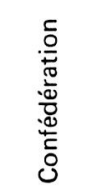 & 要 & 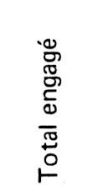 & \\
\hline \multicolumn{2}{|c|}{ ANCIENS CREDITS } & & & & & \\
\hline 1966 & $\begin{array}{l}\text { Inde (G) } \\
\text { Industrie }\end{array}$ & 31,5 & 31,5 & 63,0 & 63,0 & $\begin{array}{l}6-10 \\
11-15(2)\end{array}$ \\
\hline 1970 & $\begin{array}{l}\text { Pakistan (G) } \\
\text { Industrie }\end{array}$ & 22,5 & 22,5 & 45,0 & 45,0 & $\begin{array}{l}6-10 \\
11-15 \text { (2) }\end{array}$ \\
\hline 1973 & $\begin{array}{l}\text { Inde (G) } \\
\text { Industrie }\end{array}$ & 24,75 & 24,75 & 49,5 & 49,5 & $\begin{array}{c}7-12 \\
13-18(2)\end{array}$ \\
\hline 1977 & $\begin{array}{l}\text { Tunisie (G) } \\
\text { Installations portuaires, } \\
\text { industrie textile }\end{array}$ & 20,0 & 10,0 & 30,0 & 28,0 & $\begin{array}{r}4-10 \\
11-15\end{array}$ \\
\hline $\begin{array}{l}1979 \\
\text { TOTA }\end{array}$ & $\begin{array}{l}\text { Egypte (G) } \\
\text { Réseau électrique, } \\
\text { transports, etc. } \\
\text { AL }\end{array}$ & 45,0 & 15,0 & 60,0 & 60,0 & $\begin{array}{r}4-10 \\
11-15\end{array}$ \\
\hline
\end{tabular}




\begin{tabular}{|c|c|c|c|c|c|}
\hline \multirow[b]{2}{*}{$\begin{array}{l}\text { Pays } \\
\text { Type de crédit (1) } \\
\text { Champ d'utilisation }\end{array}$} & \multicolumn{4}{|c|}{ Montants en millions de francs } & \multirow{2}{*}{ 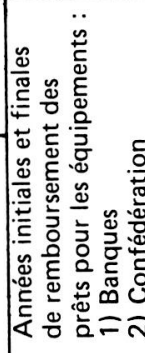 } \\
\hline & 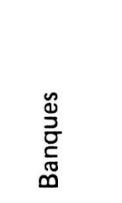 & 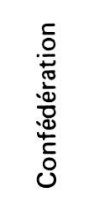 & 覀 & 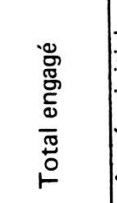 & \\
\hline \multicolumn{6}{|c|}{ CREDIT DE PROGRAMME DU 28 NOVEMBRE 1978} \\
\hline $\begin{array}{l}1979 \text { Thaïlande }(B) \\
\text { Electrification rurale }\end{array}$ & 38,25 & 12,75 & 51,0 & 37,8 & $\begin{array}{r}4-10 \\
11-15\end{array}$ \\
\hline $\begin{array}{l}1979 \text { Sri Lanka (G) } \\
\text { Télécommunications et } \\
\text { réseau électrique }\end{array}$ & 15,0 & 15,0 & 30,0 & 14,3 & $\begin{array}{r}4-10 \\
11-20\end{array}$ \\
\hline $\begin{array}{l}1980 \text { Sénégal (G) } \\
\text { Services publics : } \\
\text { agriculture, santé, etc. }\end{array}$ & 12,0 & 12,0 & 24,0 & 11,5 & $\begin{array}{r}4-10 \\
11-20\end{array}$ \\
\hline $\begin{array}{l}1981 \text { Cameroun (G) } \\
\text { Services publics : } \\
\text { eau, routes, santé }\end{array}$ & 10,0 & $\begin{array}{r}10,0 \\
(+2,0)\end{array}$ & $\begin{array}{r}20,0 \\
(+2,0)\end{array}$ & $\begin{array}{c}3,1 \\
(+0,3)\end{array}$ & $\begin{array}{r}\text { 4-10 } \\
\text { (don) }\end{array}$ \\
\hline $\begin{array}{l}1981 \text { Honduras (P) } \\
\text { Centrales hydroélectriques }\end{array}$ & 15,5 & 15,5 & 31,0 & 31,0 & $\begin{array}{r}6-17 \\
18-29\end{array}$ \\
\hline $\begin{array}{l}1981 \text { Zimbabwe : 1er accord (G) } \\
\text { Chemins de fer, } \\
\text { (locomotives électriques) }\end{array}$ & 11,475 & 7,65 & 19,125 & 19,125 & $\begin{array}{l}1-10(3) \\
11-25\end{array}$ \\
\hline $\begin{array}{c}1981 \text { Kenya (B) } \\
\text { Industrie }\end{array}$ & 10,0 & 10,0 & 20,0 & 0,0 & $\begin{array}{r}4-10 \\
11-20\end{array}$ \\
\hline $\begin{array}{l}1982 \text { Maroc }(G) \\
\text { Services publics et } \\
\text { industrie }\end{array}$ & 37,4 & 17,6 & 55,0 & 0,0 & $\begin{array}{r}4-10 \\
11-25\end{array}$ \\
\hline $\begin{array}{l}1982 \text { Zimbabwe : } \\
\text { 2ème accord (G) } \\
\text { Modernisation de } \\
\text { l'industrie }\end{array}$ & 10,0 & 10,0 & 20,0 & 0,0 & $\begin{array}{r}4-10 \\
11-15\end{array}$ \\
\hline TOTAL (+ don au Cameroun) & 159,625 & $\begin{array}{l}110,50 \\
(+2,0) \\
\end{array}$ & $\begin{array}{l}270,125 \\
(+2,0) \\
\end{array}$ & $\begin{array}{l}116,825 \\
(+0,3) \\
\end{array}$ & \\
\hline \multicolumn{6}{|l|}{ CREDIT RECEMMENT ANNONCE } \\
\hline $\begin{array}{ll}\text { 1982-Pérou (G) } \\
83 & \begin{array}{l}\text { Plusieurs projets } \\
\text { d'infrastructure }\end{array}\end{array}$ & 40,0 & 20,0 & 60,0 & 0,0 & $\begin{array}{l}\text { pas } \\
\text { encore } \\
\text { annoncé }\end{array}$ \\
\hline
\end{tabular}


1. Types de crédit : ligne de crédit générale $(G)$, ligne de crédit destiné à une branche déterminée $(B)$, ou crédit de projet $(P)$.

2. En 1977, la part déjà versée de la tranche officielle de ces crédits a été transformée en dons conformément à "I'action spéciale" convenue à l'issue de la Conférence sur la coopération économique internationale (Paris, juin 1977).

3. Alors que dans la plupart des autres cas les délais de remboursement commencent à courir au moment des tirages sur le crédit, dans ce cas le délai est compté à partir du montage et de la mise en service des équipements (au plus tard trente-trois mois après la signature de l'accord de crédit).

Sources :

- Messages du Conseil fédéral (en particulier Message du 14 décembre 1981, annexe 4).

- Rapports sur la politique économique extérieure (textes d'accords concernant l'ouverture de crédits mixtes).

- Annuaire Suisse-Tiers Monde 1981, pp. 109-110.

- DDA et OFAEE : Coopération suisse au développement : information trimestrielle concernant les mesures bilatérales de la Confédération en préparation, juillet 1982.

- Notes explicatives aux exportateurs (diffusées par les consortiums de banques).

- Renseignements communiqués par I'OFAEE.

\section{Précisions concernant les conditions financières, le champ d'utilisation et le choix des pays (1)}

\section{a) Conditions financières}

La part bancaire des crédits mixtes est prêtée aux conditions du marché tandis que la Confédération accorde des conditions préférentielles. Le résultat de l'opération est un crédit assorti d'un taux d'intêrêt moyen et de délais de remboursement prolongés. Les crédits mixtes forment par conséquent une catégorie intermédiaire, située entre l'aide publique au développement et les crédits-cadres (ou crédits de transfert) privés. Jusqu'à présent, la participation de la Confédération a varié entre 25 et $50 \%$ du montant des crédits mixtes.

\section{Conditions de la part bancaire}

Généralement, le taux d'intérêt est de $11 / 2$ à $15 / 8 \%$ supérieur au taux d'émission des obligations de caisse de cinq ou huit ans, calculé au moment des tirages sur le crédit. Le taux d'intérêt est fixé pour une période de cinq à dix ans. Pour les biens d'équipement, la durée du crédit est normalement de dix ans, avec un délai de franchise de trois ans (autre livraisons et services : cinq ans, avec un délai de deux ans et demi).

1. Les précisions s'appliquent aux crédits mixtes accordés depuis 1977.

Source : Messages concernant le financement de mesures de politique économique et commerciale au titre de la coopération internationale au développement, du 9.8.1978 et du 14.12.1981. 
Conditions de la part officielle

Sans intérêt. Pour les biens d'équipement, la durée du prêt des de dix à vingtcinq ans, avec un délai de franchise de dix ans. L'amortissement du crédit public commence donc après le remboursement du crédit bancaire. Pour les autres livraisons et les services, les conditions sont conformes à celles de la part bancaire.

\section{b) Champ d'utilisation}

II existe trois types de crédits mixtes :

- Des lignes générales de crédit destinées à toutes les branches économiques. Les livraisons pouvant être financées à l'aide du crédit figurent sur une "liste standard" comprenant une vingtaine de catégories de biens d'investissement et de services utiles au développement et pour lesquels les fournisseurs suisses sont aptes à faire des offres compétitives.

- Des lignes de crédit destinées à une ou à quelques branches économiques prioritaires.

- Des lignes de crédit destinées à un seul projet, généralement dans le domaine de l'infrastructure. II s'agit le plus souvent d'un grand projet financé par plusieurs institutions de développement ("cofinancement").

Actuellement, la plupart des crédits mixtes sont des lignes générales de crédit. Cependant, la Confédération prévoit de consacrer davantage de crédits à des cofinancements.

Voici la répartition par branches économiques des crédits mixtes engagés ou prévus actuellement :

$\begin{array}{lr}\text { - Infrastructure économique } & \\ \text { (dont énergie 40\%, transports 28\%) } & 72 \% \\ -\begin{array}{l}\text { Infrastructure sociale } \\ \text { (dont adduction d'eau 7\%) }\end{array} & 11 \% \\ -\begin{array}{l}\text { Industrie } \\ \text { (dont industrie textile 5\%) }\end{array} & 10 \% \\ - \text { Agriculture et agro-industrie } & 5 \% \\ - \text { Commerce et administration } & 2 \%\end{array}$

\section{c) Critères de choix des pays}

Trois critères se rapportent à la situation des pays destinataires et un à l'expansion des échanges avec la Suisse : 


\section{Etat de développement}

En règle générale, la Confédération accorde les crédits mixtes à des pays en développement dont l'économie est partiellement modernisée, mais qui ont un accès limité aux crédits commerciaux et qui ne peuvent pas supporter un service de la dette extérieure élevé. La Confédération n'accorde pas, en principe, de crédit mixte à des pays dont le revenu par habitant dépasse 1.000 dollars (au prix de 1978).

Politique de développement

Elle doit être conforme aux principes de la coopération suisse, c'est-à-dire viser un développement équilibré et favoriser la participation des régions et des couches de population les plus pauvres. Dans ses évaluations, I'administration fédérale se fonde sur les plans de développement des pays concernés et sur les statistiques économiques et sociales.

Capacité d'absorption

Les destinataires doivent avoir l'usage d'un crédit mixte et être à même d'en assumer les charges financières et administratives. Les crédits mixtes ne conviennent pas aux pays les plus pauvres, sans recettes d'exportations suffisantes ou surendettés. La Confédération préfère leur accorder des contributions non remboursables, dans les limites des budgets de l'aide publique au développement.

\section{Expansion des échanges avec la Suisse}

Les crédits mixtes étant liés à l'achat de marchandises suisses, le pays destinataire doit effectivement avoir besoin de la gamme des produits diffusés par la Suisse. Pour leur part, les exportateurs doivent avoir intérêt à l'expantion des échanges avec ce pays et être prêts à lui faire des offres compétitives. Sinon, le crédit inciterait ce pays à importer des marchandises onéreuses, ou bien le crédit resterait inutilisé.

\section{L'effet sur les exportations suisses de biens d'équipement (données par pays et explication des calculs)}

L'effet des crédits mixtes sur les exportations suisses est un phénomène complexe car les situations des pays destinataires et les circonstances de l'octroi des crédits sont très diverses. Chaque cas mériterait une étude spécifique. II est néanmoins intéressant de tenter une comparaison générale entre les crédits mixtes et les exportations actuelles de biens d'équipement vers les pays emprunteurs. Le tableau suivant concerne les crédits accordés depuis 1977. 


\section{CREDITS MIXTES ET EXPORTATIONS DE BIENS D'EQUIPEMENT}

\begin{tabular}{|l|c|c|c|c|}
\hline \multirow{1}{*}{ Pays } & $\mathrm{a}$ & $\mathrm{b}$ & $\mathrm{c}$ & $\mathrm{d}$ \\
\cline { 2 - 5 } & $\begin{array}{c}\text { Montants des } \\
\text { crédits mixtes }\end{array}$ & $\begin{array}{c}\text { Exportations } \\
\text { annuelles } \\
\text { d'équipements } \\
\text { (moyenne } \\
1980-1981)\end{array}$ & $\begin{array}{c}\text { Années } \\
\text { d'exportations } \\
\text { couvertes par } \\
\text { le crédit mixte }\end{array}$ & $\begin{array}{c}\text { Déboursements } \\
\text { annuels en \% } \\
\text { des exportations } \\
\text { annuelles } \\
\text { d'équipements }\end{array}$ \\
\cline { 2 - 5 } & En Mio Fr. & En Mio Fr. & $\mathrm{a} / \mathrm{b}$ & $\frac{\mathrm{a} / 7}{\mathrm{~b}} \cdot 100$ \\
\hline Tunisie & 30,0 & 26,7 & 1,1 & 16 \\
Egypte & 60,0 & 192,7 & 0,3 & 4 \\
Thaïlande (1) & 51,0 & 44,2 & $1,2(1)$ & $16(1)$ \\
Sri Lanka & 30,0 & 7,1 & 4,2 & 61 \\
Sénégal & 24,0 & 1,9 & 13,0 & 185 \\
Cameroun & 20,0 & 14,0 & 1,4 & 20 \\
Honduras (2) & 31,0 & 1,7 & $18,5(2)$ & $264(2)$ \\
Zimbabwe (3) & 39,1 & 20,0 & $2,0(3)$ & $28(3)$ \\
Kenya & 20,0 & 23,4 & 0,9 & 12 \\
Maroc & 55,0 & 31,0 & 1,8 & 25 \\
Pérou & 60,0 & 67,3 & 0,9 & 13 \\
Ensemble & 420,1 & 430,0 & 1,0 & 14 \\
\hline
\end{tabular}

1. La comparaison avec les exportations de biens d'équipement est seulement valable en partie. Le $60 \%$ du crédit est réservé pour la prestation de services dans le cadre d'un seul programme (électrification rurale).

2. Le crédit sert au financement d'un seul projet (centrale hydroélectrique). Les commandes ont été attribuées à la suite d'un appel d'offres international.

3. Le montant concerne deux accords de crédit. Le premier, qui s'éléve à 19,125 millions de francs, concerne un seul projet (locomotives électriques). Les commandes ont été attribuées à la suite d'un appel d'offres international.

\section{Explication des colonnes :}

a) Montants des crédits mixtes : total des parts bancaire et publique.

b) Exportations annuelles de biens d'équipement (moyenne 1980-1981) :

- Il s'agit des exportations indiquées dans la Statistique annuelle du commerce extérieur de la Suisse. La correspondance avec la "liste standard des biens et services pouvant être financés par les crédits mixtes" est partielle : la statistique ne comprend pas certaines prestations figurant sur la liste (biens de catégorie spéciale et services) tandis que cette dernière ne comprend pas tous les biens d'équipement indiqués dans la statistique. 
- Dans la majorité des cas, les versements faits au titre des crédits mixtes sont encore faibles (ou nuls). Ils n'ont, par conséquent, pas eu beaucoup d'influence sur le volume des exportations en 1980 et 1981.

c) Années d'exportations couvertes par le crédit :

- Dans quelques cas (Thaïlande, Honduras et Zimbabwe notamment), les données sont difficilement comparables (voir notes 1, 2 et 3 sous le tableau).

- Deux facteurs ne sont pas pris en considération : le rapport précis entre les prêts et la valeur des livraisons (le financement s'élève généralement à $85 \%$ de cette dernière);

la hausse des prix à l'exportation pour ces prochaines années (entre 1979 et 1981, par exemple, le prix des équipements a augmenté de 2,9\% par année selon la statistique du commerce extérieur).

d) Déboursements annuels en \% des exportations annuelles d'équipements :

- L'administration fédérale estime que la période moyenne de déboursement des crédits ne devrait pas excéder sept ans (sans tenir compte de versements minimes s'étalant éventuellement sur plusieurs années au début et à la fin des périodes). Les déboursements annuels (montant des crédits mixtes divisé par sept) ont été calculés sur la base de cette prévision.

- Les exportations annuelles de biens d'équipement retenues ici correspondent à la moyenne des années 1980-1981 (colonne b).

\section{Sources :}

- Point 1 : "Crédits mixtes accordés à ce jour".

Statistique annuelle du commerce extérieur de la Suisse, 1980 et 1981, Vol. I. 


\section{NOTES DES CHAPITRES I A IV}

Le lecteur trouvera les références complètes des textes dans la bibliographie.

\section{LE DESEQUILIBRE DES ECHANGES SUISSE-TIERS MONDE}

1. Par souci de concision, nous avons limité la citation de chiffres. Des statistiques détaillées se trouvent à la fin de l'Annuaire. Le lecteur y trouvera aussi la définition des catégories statistiques utilisées et des remarques concernant l'interprétation des chiffres.

2. Pour les raisons indiquées plus haut, le montant de l'excédent n'est malheureusement pas connu. Selon les estimations de R. Gerster, les recettes de la Suisse ont presque atteint le double des dépenses en 1974. Voir "Schweizerisches Volkseinkommen und Dritte Welt", pp. 33-71.

3. Ph. BERBERAT et H. STETTER, “Relations économiques Suisse-Tiers Monde : Bilan, perspectives, propositions de changement".

4. A la fin des années soixante, la part des industries extractives (agriculture, mines et pétrole) représentait encore presque la moitié des investissements directs étrangers dans le Tiers Monde.

5. Réponse du Conseil fédéral au postulat Generali du 19.3.1981 : “Aide au développement. Conséquences économiques", pp. 117-118. Le montant de 337 millions de francs comprend en particulier la contre-valeur des livraisons de biens et services suisses aux bénéficiaires de crédits accordés par les banques internationales de développement soutenues par la Confédération.

\section{LA COOPERATION AU DEVELOPPEMENT DANS LE CONTEXTE DE LA POLITIQUE ECONOMIQUE EXTERIEURE}

1. Message du 19.3.1973, p. 4.

La présentation de la coopération suisse au développement et l'analyse de ses objectifs, de ses principes et de ses formes font l'objet, dans cet Annuaire, d'un article de Jacques Forster : “La coopération de la Suisse au développement : politique et réalisations".

2. Pour une analyse des buts de la coopération suisse au développement, voir l'article de Jacques Forster, point 2.2. "La promotion du développement".

3. Rapport concernant les répercussions des récents événements économiques mondiaux..., 22.1.1975, pp. 27-30. Voir aussi I'article de Jacques Forster, sous le point 2.1. : "La défense des intérêts de la Suisse".

4. Message du 9.8.1978, pp. 4-15.

5. Idem, p. 15

6. Idem, pp. 19 et 20.

7. Idem, p. 5.

8. Idem, p. 20.

9. Idem, p. 26. On trouve aussi ce type de comparaison dans d'autres messages sur la coopération au développement.

10. Message du 19.3.1973, p. 27. 
11. Deuxième alinéa de l'article 1er de la loi fédérale sur la garantie contre les risques à l'exportation (du 26.9.1958), inséré le 10 octobre 1980.

12. Voir réponse du Conseil fédéral à la question ordinaire Blunschy (No 81.769) du 14.12.1981.

13. Chiffres indiqués dans le Dix-huitième rapport sur la politique économique extérieure et dans la documentation de presse diffusée par le Service de la garantie contre les risques à l'exportation de l'OFAEE. Voir aussi dans la partie statistique de cet Annuaire, tableau 2.4.B.2.

\section{MESURES ECONOMIQUES ET COMMERCIALES}

1. Feuille fédérale, 1978, Vol. II, pp. 1765 ss.

2. Ordonnance concernant la coopération au développement et l'aide humanitaire internationales, du 17.12.1977 (RO 1978).

Selon l'article 2 de l'ordonnance, alinéas 2 et 3, “lorsqu'un office a le droit de se prononcer, l'office fédéral compétent n'est pas lié par I'opinion de cet autre office. Lorsque I'office fédéral agit d'entente avec un autre, il ne peut prendre de décision sans l'accord de celui-ci".

3. Les parties descriptives de chaque rubrique proviennent principalement des Messages sur le financement de mesures de politique économique et commerciale, du 9.8.1978 et du 14.12.1981.

4. Message du 25.8.1981, p. 46. Pour la présentation des préférences tarifaires, voir aussi Annuaire Suisse-Tiers Monde 1981, pp. 42 à 49, et 1982, partie "Revue", chap. "Négociations internationales", point 6.3.1.

5. DDA, La coopération suisse au développement : Bulletin de statistiques, 1980, tableau 1.1 et La DDA en 1981, Annexe III. Les contributions accordées au Portugal et à la Turquie ne font pas partie des mesures prévues par l'arrêté fédéral du 28.11. 1978. La Confédération finançait déjà des mesures économiques et commerciales auparavant. Leur coût a seulement été estimé à partir de 1978. II a atteint, cette année-là, 13,1 millions de francs $(4,2 \%$ de l'aide publique au développement).

6. Message du 14.12.1981, p. 37. Cette part peut aussi se calculer à partir du tableau récapitulatif des crédits de programme indiqué aux pp. 36-37. Considérons les moyennes annuelles maximales d'engagement des dépenses (montant des crédits de programme divisé par la durée minimale d'utilisation) :

- Coopération technique et aide financière Mio $\mathrm{Fr}$. $\quad \%$

- Aide humanitaire

$550 \quad 69$

- Mesures économiques et commerciales

- Banques régionales de développement $120 \quad 15$ (participation au capital : montant à libérer)

$117 \quad 15$
TOTAL

\begin{tabular}{rr}
11 & 1 \\
\hline 798 & 100 \\
\hline
\end{tabular}

Comme pour les autres crédits de programme, la part prévue pour les mesures économiques et commerciales représente un ordre de grandeur. Le pourcentage précis est susceptible de varier d'une année à l'autre.

7. Message du 9.8.1978, p. 5.

8. Idem, pp. 3-4. 
9. Message du 14.12.1981, pp. 11-13. (Les citations se trouvent respectivement aux pages $11-12,12,11$ et 13.$)$

10. Message du 9.8.1978, p. 20.

11. Message du 14.12.1981, pp. 11-13. (Les citations se trouvent respectivement aux pages 12,13 et 11.$)$

12. Message du 9.8.1978, p. 26.

13. Pour la présentation de cette intervention, voir Message portant midification de l'arrêté fédéral sur la collaboration de la Suisse aux mesures monétaires internationales, du 30.5.1979.

14. Idem, p. 6. La "facilité Vitteveen" est entrée en vigueur en février 1979. La Banque nationale suisse y participe à raison de 650 millions de Droits de tirage spéciaux. Cette opération n'est pas couverte par la garantie de 2 milliards de francs mentionnée plus haut.

15. Message du 14.12.1981, p. 20.

16. La présentation détaillée de l'action de la Confédération se trouve dans les Messages du 9.8.1978 et du 14.12,1981 (cités) et dans le Message sur des mesures commerciales et des mesures relatives aux produits de base dans le cadre de la coopération au développement, du 25.2.1981.

17. Voir partie "Revue" de cet Annuaire, chapitre "Négociations internationales", point 6.2.1.

18. Lorsque les fournisseurs ont un pouvoir de négociation suffisant, la Suisse accepte cependant de conclure des accords de livraison à long terme. C'est par exemple le cas pour l'uranium, le gaz naturel et le pétrole brut. Le 20 août dernier, la Carbura, association privée regroupant la plupart des importateurs suisses de pétrole brut, a créé le consortium "Rohoel-Plattform" qui pourra se présenter en tant qu'acheteur unique face aux Etats producteurs qui veulent traiter avec un seul interlocuteur par pays de destination (de préférence avec I'Etat ou avec une compagnie publique). Vu I'abondance actuelle de l'offre de pétrole, ce consortium n'interviendra pas dans l'immédiat. A l'avenir cependant, en cas de difficultés d'approvisionnement notamment, il pourrait faciliter la recherche de nouveaux fournisseurs. Journal de Genève, 24.8.1982. (Le consortium servira peut-être aussi à rééquilibrer la balance commerciale avec des pays pétroliers - comme l'Irak en particulier - qui sont d'excellents clients de la Suisse, mais auxquels notre pays n'achète actuellement pas de pétrole pour la raison susmentionnée.)

19. E. ROETHLISBERGER, "La politique des produits de base vue par la Suisse", p. 22. ( $L$ 'ambassadeur $E$. Roethlisberger est délégué aux accords commerciaux et responsable des questions du développement à l'OFAEE.)

20. Le Conseil fédéral prévoit d'attribuer environ 20 millions de francs pour les produits de base sur le nouveau crédit de programme concernant les mesures de politique économique et commerciale. Ce montant peut être augmenté le cas échéant si les sommes prévues pour d'autres mesures ne sont pas dépensées. Le crédit sera ouvert pour une durée d'au moins trois ans. Cf. Message du 14.12.1981, p. 36.

21. De tels contrats devraient être conclus dans l'attente de mesures efficaces sur le plan multilatéral. Dans une perspective globale, les conventions bilatérales d'approvisionnement ont en effet aussi certains inconvénients : manque de transparence des contrats, conditions plus favorables obtenues par les producteurs ou les consommateurs détenteurs de positions dominantes sur le marché, etc. Voir E. Roethlisberger, "La politique des produits de base vue par la Suisse", p. 24. Les inconvénients cités par l'auteur nous apparaissent cependant inférieurs à ceux que les pays en développement conti- 
nuent à subir dans le cadre des structures actuelles des marchés des matières premières.

22. A. NYDEGGER, Förderung von Importen aus Entwicklungs/ändern in die Schweiz, 1. Teil, S. 40-43.

23. Voir précisions dans la partie "Revue" de cet Annuaire, chapitre "Politique économique extérieure", point 9 . Depuis le 1er juillet 1982, les trente et un pays les moins développés bénéficient de l'exemption totale des droits de douane sur les produits industriels. Pour l'explication du schéma suisse des préférences tarifaires, voir Annuaire Suisse-Tiers Monde 1981, pp. 42-49.

24. H. STETTER, T. VATTER, Importförderung : entwicklungspolitisch begriffen, S. 36-37, 58-59, 95-98. Résumé français : La promotion des exportations (dans une optique de politique de développement). pp. 7-9.

25. La Confédération soutient les activités du Centre du commerce international (CNUCED/GATT) à Genève. Le Comptoir suisse et la Foire suisse d'échantillons accueillent chaque année quelques pays en développement, grâce aux subsides fédéraux. Il est aussi prévu de faire appel aux œuvres suisses d'entraide. La Confédération appuie déjà un projet de l'Organisation Suisse-Tiers Monde (OS3) consistant à promouvoir sur le marché suisse des produits artisanaux en jute fabriqués au Bangladesh: voir l'article de F. de Morsier dans cet Annuaire.

26. Le montant total prévu pour la promotion commerciale s'élève à 10 millions de francs. II peut être augmenté le cas échéant si les sommes prévues pour d'autres mesures ne sont pas dépensées. La durée du crédit est d'au moins trois ans. Cf. Message du 14. 12.1981 , p. 36.

27. La réflexion menée par R. STRAHM sur la promotion commerciale et sur la création d'un centre chargé de cette tâche reste très actuelle. Voir : L'ambiguïté des échanges avec le Tiers Monde, la Suisse peut-elle favoriser le développement par une promotion de ses importations en provenance du Tiers Monde?

28. Le Conseil fédéral a d'ailleurs retenu cet objectif. Voir Message du 9.8.1978, p. 26. Quelques projets du Centre du commerce internatinal (CNUCED/GATT) soutenus par la Confédération favorisent aussi les échanges entre les pays en développement.

29. Voir partie statistique de cet Annuaire, tableau 2.4.A.2. "Investissements directs nets par groupes de pays classés selon le produit national brut par habitant (1979)".

30. Voir partie "Revue" de cet Annuaire, chapitre "Politique économique extérieure", point 8.

31. La Confédération a l'obligation de tenir compte des principes fondamentaux de la politique de développement lors de l'octroi de garanties concernant des exportations vers les pays en développement les plus défavorisés. (Voir plus haut, chap. II.)

32. Voir partie "Revue" de cet Annuaire, chapitre "Politique économique extérieure", point 7.

\section{L'ASSOCIATION DE CAPITAUX PUBLICS ET DE CAPITAUX PRIVES : LE CAS DES CREDITS MIXTES}

1. CREDIT SUISSE, Manuel de financement des exportations, pp. 53-54.

2. H. BUEHLER, "Les accords de crédit entre la Confédération suisse et I'Inde". 1967. L'auteur était alors vice-directeur de la Division du commerce, au Département fédéral de l'économie publique.

3. Idem, p. 68. 
4. Messages concernant l'ouverture d'un crédit de transfert au gouvernement de la République de l'Inde, du 4.5.1965 et à la République Islamique du Pakistan, du 28.5.1969. Concernant I'Inde, voir aussi l'article de H. Bühler.

5. Pour les prêts récents, voir Annuaire Suisse-Tiers Monde 1981, pp. 118-120, 230 232 et Annuaire Suisse-Tiers Monde 1982, p. 65.

6. Message concernant l'aide à la Turquie, 28.8.1968, pp. 10 et 14.

7. Voir par exemple accords avec le Honduras ou avec le Zimbabwe, dans le Dix-huitième rapport sur la politique économique extérieure, du 25.1.1982, pp. 100 et 108.

8. Bulletin officiel de l'Assemblée fédérale, 23.6.1982, pp. 892-900.

9. Idem. Déclaration de Y. Jaggi, au nom du Groupe socialiste, pp. 896-897.

10. Idem. Rapports de J. Risi et de G. Coutau, au nom de la Commission des affaires économiques, pp. 894 et 895.

11. Idem. Déclaration de C. Blocher, au nom du Groupe de I'Union démocratique du centre, p. 898. L'orateur affirma aussi que le contrôle de l'efficacité des crédits mixtes était bien mieux assuré que celui des crédits destinés par exemple à la coopération technique ou à l'aide humanitaire.

12. Idem. Rapport de J. Risi, au nom de la Commission des affaires économiques, p.893.

13. Pour un prêt de vingt ans, sans intérêt, remboursable semestriellement après dix années d'attente, l'élément-don représente environ $65 \%$ du montant nominal du crédit, compte tenu d'un taux d'actualisation de $7 \%$ (ce taux est plus réaliste pour les crédits suisses à l'exportation que le taux forfaitaire de $10 \%$ retenu par le Comité d'aide au développement de l'OCDE).

14. Cette amélioration va dans le sens des efforts du Comité d'aide au développement de I’OCDE, qui souhaite que les crédits mixtes accordés par les pays membres ne soient pas des crédits à l'exportation déguisés.

15. Tel n'étant manifestement pas le cas pour de nombreux pays du Tiers Monde, l'administration fédérale étudie la possibilité $d$ 'accepter le remboursement de la part publique en monnaie locale. Message du 14.12.1981, p. 30.

16. Voir "Conditions attachées à I'aide", Message du 23.11.1977, pp. 44-48.

17. Le second objectif est particulièrement significatif lorsque les firmes suisses participent à un projet de la Banque mondiale : depuis le rejet en 1976, lors d'un vote populaire, d'un prêt de 200 millions de francs à l'Association internationale de développement, la Suisse ne participe plus aux reconstitutions du capital de celle-ci. Cette abstention est de moins en moins admise sur le plan international.

18. Le Groupe radical, le Groupe socialiste et le Groupe du parti du travail et du parti socialiste autonome manifestèrent ce souhait lors de la séance du 23.6.1982 du Conseil national. B. Hunziker, intervenant au nom du parti radical, insista sur ce point. II se demanda notamment si I'Inde et le Maroc méritaient d'être soutenus. La première s'offre le luxe de l'arme atomique et le second est en guerre avec un voisin pacifique. L'orateur demanda aussi s'il convenait d'aider des pays qui bloquent des avoirs suisses. Bulletin officiel de l'Assemblée fédérale, 23.6.1982, p. 898.

19. Message du 14.12.1981, p. 32.

20. Les poúrcentages se rapportent aux montants des exportations suisses figurant dans la partie statistique de cet Annuaire (cf. tableaux 1.1. et 1.2.).

21. Message du 14.12.1981, p. 33.

22. G. Coutau, rapporteur de la Commission des affaires économiques du Conseil national, s'exprimant sur les mesures de politique économique et commerciale au titre de la coopération internationale au développement. Bulletin officiel de l'Assemblée fédérale, 23.6.1982, p. 895. 
23. L'accord de crédit conclu avec le Zimbabwe pour la construction de locomotives électriques prévoit une petite part pour des fournisseurs locaux. 


\section{BIBLIOGRAPHIE}

\section{Principaux ouvrages et documents cités}

Assemblée fédérale - Loi sur la coopération au développement et l'aide humanitaire internationales, du 19.3.1976.

BERBERAT Ph. et STETTER H., "Relations économiques Suisse - Tiers Monde : Bilan, perspectives, propositions de changement", Nyon, Fondation internationale pour un autre développement, Dossier FIPAD no 10, août 1979 (article et document ronéoté).

BERWEGER G. - Investition und Legitimation. Privatinvestitionen in Entwicklungsländern als Teil der schweizerischen Legitimationsproblematik. Diessenhofen, Verlag Rüegger, 1977.

BLUNTSCHLI F. - Zu den Beziehungen zwischen schweizerischer Aussenwirtschafts- und Entwicklungspolitik. Versuch einer Klärung und Orientierung im bestehenden Weltwirtschaftssystem. Adliswil, Institut für Sozialethik des SEK, 1980.

BUEHLER H. - "Les accords de crédit entre la Confédération suisse et I'Inde", Revue économique et sociale, 1967, numéro spécial, pp. 67-75.

\section{CONSEIL FEDERAL :}

- Message à l'appui d'un projet de loi sur la coopération au développement et l'aide humanitaire internationales, du 19.3.1973.

- Messages concernant la coopération technique et l'aide financière en faveur des pays en développement, des 27.9.1976, 23.11.1977 et 9.7.1980.

- Messages concernant le financement de mesures de politique économique et commerciale au titre de la coopération internationale au développement, des 9.8.1978 et 14.12.1981.

- Message sur des mesures commerciales et des mesures relatives aux produits de base dans le cadre de la coopération au développement, du 25.2.1981.

- Rapport concernant les répercussions des récents événements économiques mondiaux sur la contribution suisse à la coopération internationale au développement, du 22.1.1975.

- Rapports sur la politique économique extérieure, parution semestrielle.

- Réponse au postulat Generali (81.375) du 19.3.1981 : “Aide au développement. Conséquences économiques". Dans : Dix-huitième rapport sur la politique économique extérieure, du 25.1.1982, annexe 6, pp. 115-128. 
CONSEIL NATIONAL - "Coopération au développement : mesures commerciales". Procès-verbal du débat du 23.6.1982, Bulletin officiel de l'Assemblée fédérale, 1982, pp. 892-900.

CREDIT SUISSE - Manuel de financement des exportations, Cahiers du Crédit Suisse, no 40, 1979, 3e éd.

FORSTER J. - “La coopération de la Suisse au développement : politique et réalisations", Annuaire Suisse-Tiers Monde 1982, Genève, IUED, 1982.

GERSTER R. - "Schweizerisches Volkseinkommen und Dritte Welt", Aussenwirtschaft, März 1976, S. 33-71.

HAMMER P. - Funktion und Verfassungsmässigkeit der schweizerischen Entwicklungspolitik (Dissertation Universität Freiburg), Grenchen, 1974.

NYDEGGER A. (Leitung) - Förderung von Importen aus Entwicklungs/ändern in die Schweiz. Vorstudie im Auftrag der Direktion für Entwicklungszusammenarbeit und humanitäre Hilfe des Eidgenössischen Politischen Departements. St Gallen, Mai 1979. 1. Teil.

REHSCHE G. - Schweizerische Aussenwirtschaftspolitik und Dritte Welt. Ziele und Instrumente - Exportförderung kontra Entwicklungspolitik? Adliswil, Institut für Sozialethik des SEK, 1977.

ROETHLISBERGER E. - "La politique des produits de base vue par la Suisse", Entwicklung-Développement, no 9, 1981, pp. 22-25.

STETTER H. und VATTER T. - Importförderung : entwicklungspolitisch begriffen. Sonceboz, Import- und Informationsstelle für Waren aus Entwicklungsgebieten (OS3). 1981. Résumé français : La promotion des exportations (dans une optique de politique de développement).

STRAHM R. - L'ambiguïté des échanges avec le Tiers Monde. La Suisse peut-elle favoriser le développement par une promotion de ses importations en provenance du Tiers Monde? Berne et Lausanne, Institut d'éthique sociale de la FEPS, Etudes et Rapports no 15-16, nov. 1975. 